\title{
Influential Article Review - Objective or Interrelated Strategies: Inbound Marketing vs. Outbound Marketing
}

\author{
Malikah Holden \\ Latisha Salinas \\ Jonah Zhang
}

This paper examines digital marketing. We present insights from a highly influential paper. Here are the highlights from this paper: This article tends to explain the differences between the Outbound Marketing strategy and the Inbound Marketing strategy, by offering a comparison of these techniques used by these two approaches. This paper aims to determine the impact of the "new marketing strategies" on the existent ones, and clarifies if these two strategies are complementary or independent for a company operating in a highly competitive environment. For our overseas readers, we then present the insights from this paper in Spanish, French, Portuguese, and German.

Keywords: digital marketing, inbound marketing, marketing strategy, online marketing, outbound marketing

\section{SUMMARY}

- Outbound marketing is interruption-based marketing, perceived as the traditional model of product promotion in which people have to stop what they are doing to pay attention to the marketing message or deal with it in some other way.

- The difference between inbound and outbound marketing is constantly challenged. Inbound marketing has now experienced growth compared to outbound marketing which is declining. In addition, the investments in the channels of inbound marketing have come to increase significantly, compared to other channels of outbound marketing that are now either stagnant or are experiencing slow growth.

- Volpe suggests that the difference between the incoming and the outgoing is artificial and meaningless. Moreover, he proposes a cross between the strategies of the incoming and outgoing marketing, defined as a gray zone.

- Inbound Marketing creates Top of Mind Preference, while Outbound Marketing is mainly focused on Top of Mind Awareness. With inbound content marketing programs, you can move beyond simple awareness to generate preference and implicitly brand addiction, this goal can be achieved through the positioning of a brand as the preferred choice.

- Inbound Marketing's great challenge is to get the consumer to find the company online, because of the highly competitive keyword space. 
- Indeed, the mix of outbound and inbound marketing strategies can work most effectively and reach the most amount of people when done hand-in-hand

- Nevertheless, Inbound Marketing is primarily content marketing and its efficiency is superior to the Outbound Marketing efficiency because inbound aims to identify the target customers and adapt the communication, however it allows a better targeting of the audience and a better adaptation of the company's communication mechanisms.

\section{HIGHLY INFLUENTIAL ARTICLE}

We used the following article as a basis of our evaluation:

Dakouan, C., Benabdelouahed, R., \& Anabir, H. (2019, January 29). Inbound Marketing vs. Outbound Marketing: Independent or Complementary Strategies. Expert Journal of Marketing, 7(1), 1-6.

This is the link to the publisher's website:

https://marketing.expertjournals.com/23446773-701/

\section{INTRODUCTION}

The development of marketing strategy has undergone a valuable evolution, with the existence of the digital communication and social networks, the content creation has been changed. Several years ago, the companies were using the traditional strategy, as sending a message to an audience, in order to push them towards their company or product, in opposite to the development of the new marketing strategies, namely Inbound Marketing, a new way to pull the audience towards the company, by creating more innovative content.

The State of Inbound Marketing (Hubspot, 2013) describes Outbound Marketing or traditional marketing as a technique used by firms in order to attract a large public, hoping to get a feedback. Using the outbound strategy, mainly based on the diffusion of the message and publicity, without studying the target, in order to receive an effective response and generate more sales and incomes.

Outbound marketing is interruption-based marketing, perceived as the traditional model of product promotion in which people have to stop what they are doing to pay attention to the marketing message or deal with it in some other way (Rancati et al., 2015). Outbound marketing is also a strategy in which a business advertises its products and services by presenting information to consumers even if they are not looking for those products or services (Goodwin, 2013). However, this strategy is no longer effective in getting the message across and making traffic in the web world, that is why companies, nowadays, tend to explore new strategies such as Inbound Marketing approach in order to get more results from the internet.

In the first second section of this paper, we will define inbound marketing and get an overview of the emergence of this concept. In the third section, we will explain the components of inbound marketing. In fourth section, we will compare Outbound and Inbound Marketing in order to know if they represent independent or complementary strategies.

\section{DEFINITIONS OF INBOUND MARKETING}

Inbound marketing is the strategy of connecting with potential customers through materials and experiences they find useful. Using media like blogs and social networking, marketers hope to entertain and inform viewers with content they seek by themselves (Marketing-Schools.org, 2016).

On other hand, inbound marketing can be explained as a form of marketing that requires a very careful targeting of the audience and a custom communication via high quality content (Patrutiu-Baltes, 2016).

Moreover, Venisse (2016) defined inbound marketing as a way to become its own media in order for the audience to convert more easily into customers. The implementation of the global inbound marketing strategy requires the respect of certain essential steps (Venisse, 2016). The first step is to develop its own 
media and build a qualified audience. For a company that sets up this strategy, it is important to offer interesting content on its site, its blog or its social network page, to attract and sustain an audience.

Also, Schwebel (2017) suggests that inbound marketing has the potential to develop a brand to become its own medium, that is to say, to offer content that is intelligent and relevant enough to build an audience, instead of intrusive ads (Dabi-Schwebel, 2017).

Cantin (2014) defines inbound marketing is a digital marketing strategy aimed at bringing the customer to the company rather than picking him up. The prospect is attracted by relevant content positioned according to phases of maturity of purchases and levels of commitment. Therefore, in this line of thoughts, we could also talk about the acquisition marketing (Cantin, 2014).

We define inbound marketing also as a digital marketing strategy whose general idea is to continuously attract potential prospects with quality content. To do this, Inbound Marketing uses a four-step methodology: Attract, Convert, Transform, and Retain (InboundValue.com, 2018).

Moreover, as Rouse (2014) proposed, inbound marketing can be defined "as a technique that focuses on bringing consumers or leads via relevant content, so that consumers search for the company instead of the opposite. The concept of inbound marketing make tasks easier for the consumers who want relevant goods, products or services" (Rouse, 2014).

Hubspot (2018) defines Inbound Marketing as a strategy that attracts customers with relevant and useful content and adds value at every step of the customer-shopping journey. With inbound marketing, potential customers find their way through channels such as blogs, search engines and social media (Hubspot, 2016). Unlike Outbound Marketing, Inbound Marketing does not need to fight for the attention of potential customers. By creating content that addresses the issues and needs of ideal customers, the brand attracts qualified prospects and builds trust and credibility. Hence, inbound marketing is a systemic approach that allows each brand to become its own media, create an audience and convert it into prospects and then customers. A whole process works well on the details while eliminating any failure (Dabi-Schwebel, 2017).

\section{EMERGENCE OF THE INBOUND MARKETING}

The term « Inbound» was used by Brian Halligan (Halligan et al., 2009). The term inbound refers to attracting and convert individuals to lasting customers.

Corresponding to the creators of inbound marketing Brian Halligan and Dhamesh Shah, the needs for this innovative concept was created from the fact that people are sick and tired of being interrupted with traditional outbound marketing messages. Moreover, the marketing tactics used successfully in the past didn't produce the desire results anymore (Halligan et al., 2009).

It all started when Shah started a blog on startups and entrepreneurship, then, he noticed that his blog gained a massive traffic more than websites owned by companies with professional marketing crews and big budgets. After a lot of analysis and discussions, they came out with a simple observation that "People did not want to be interrupted by marketers or harassed by salespeople, they want to be helped" (Halligan and Shah, 2014).

The main idea of inbound marketing is using marketing techniques that will try to pick up the interest of customers, by sharing creative and useful content that it is shared through multiple online channels, including search engines and social media.

Even if inbound marketing was first introduced in 2005 by Halligan and Shah, it is actually not a new concept, it is similar to the marketing method named "Content marketing" and it can also be associated to "Storytelling marketing", namely a technique used way before the emergence of the internet (Pulizzi, 2012).

Moreover, content marketing is defined as marketing process that aims to develop relevant content in order to attract and engage a target audience, thus, getting a profitable consumer action (Pulizzi, 2012).

Also, content marketing must include strategic planning, content creation and distribution for multiple stages of the buying cycle to multiple customers, means that a complete content marketing strategy should incorporate the Inbound marketing principle (Pulizzi, 2011). 
One of the inbound marketing's techniques known as the search engine optimization (SEO) was practiced for a long time before the concept of content marketing, and SEO is mostly used to increase the number of visitors that come from search engines.

The keywords were considered the most important element to achieve high ranking, then, with time and after the introduction of many updates that were included, such as Panda in 2011, Penguin in 2012 and the Hummingbird update in 2013 (Wainwright, 2014), this importance of keywords decreased. Also, because of these updates, SEO becomes a very difficult task. From here, inbound marketing comes in place, according to Rand Fishkin, SEO is a tactic, and inbound marketing is a strategy.

Email marketing also refers to one of the important elements of inbound marketing based on sending newsletter, articles or others, via an e-mail. It is a type of marketing based on the permission given by the visitors, is it also known as permission marketing, namely a concept that was developed by Seth Godin (Godin, 1999).

\section{COMPONENTS OF INBOUND MARKETING}

\section{Content Marketing}

Although content marketing has remained in the shadow of traditional marketing given the lack of technology at that time, it has managed today to establish itself as an effective strategy especially with the rise of new technologies.

Chaffey and Al (2000) define content as a text and graphic information design that forms a web page. Good content is a keystone to attract customers to a website and retain their interest while provoking repeat visits. Content marketing can also be the company's intranet content or the company's internet content, including databases and various types of knowledge (Chaffey and Al, 2000).

For their part, Pulizzi and Barret (2009) also define content marketing as a marketing technique that consists of creating, publishing and distributing relevant, useful and valuable content to get profits from the consumers. Content marketing tends to have media rather than renting it. It is defined as a marketing process that attracts and retains customers by constantly creating content that aims to change or evolve consumer behavior (Pulizzi, J. and Barrett, 2009).

Handley and Chapman (2010) argue that content marketing is all that an individual or organization creates or shares to tell their stories. The content is conversational, human and does not constantly seek to sell. It must be a state of mind that is both timid and encouraged (Handley and Chapman, 2010).

Gunelius (2011) defines content marketing as a process of direct and indirect promotion of a company or brand through the benefit of a text, video or audio content at a time, in online and offline environments. It can appear in long-form (blogs, articles, e-books and sound), in short-form (Twitter, Facebook, images ...), or in conventional form, (content sharing via Twitter or participate in an active discussion via comments on blog posts or through online forums) (Gunelius, 2011).

Staler (2014) explains that content marketing is a dynamic technique aimed at attracting customers, it is a process of sharing expertise and knowledge in order to attract potential customers and establish a relationship with them (Slater, 2014).

Ramos (2014) defines content marketing as a high-quality marketing technique, relevant content that aims to be educational and mobilizing to acquire the target audience (Ramos, 2014).

Kotler et al. (2017) define content marketing as a marketing approach that consists of creating, organizing, distributing and amplifying interesting, relevant and useful content for a clearly defined audience group to create content conversations. Content marketing is also another form of branding and branded journalism that creates closer links between brands and customers. Ultimately, content marketing is about planning, creating, promoting, and monitoring performance for a targeted audience, always with the goal of customer satisfaction and revenue generation in mind (Kotler et al., 2017).

\section{Social Media Marketing}

The characteristics of social media and their immense popularity have revolutionized commercial practices such as advertising and promotion. 
Social networks are original spaces. They can give rise to conventional top-down communication companions (one to many), but also change this vertical and unilateral communication towards a dialogue between the user and the brand (one to one) or even more between the Internet users themselves same (many to many).

However, consumers are less and less interested in traditional advertising, which puts enormous pressure on marketers. The challenge lies in spreading complex information through messages without overwhelming consumers. The attitude of today's consumers has changed profoundly, the time of appreciation and reading of message has diminished a lot. The average attention span of humans has decreased from 12 seconds in 2000 to 8 seconds in 2013. Even more, this attention is destined to diminish in the future, because of the massive and overwhelming volume of messages constantly bombarding connected mobile devices that require immediate consumer attention (Kotler et al., 2017).

Social networks have played a very important role in these changes. In the past, consumers were limited to commercials generated on traditional media because they did not have much choice. Today, with the advent of social networks, consumers have a wealth of content generated by businesses or even by other consumers that they find more credible and interesting. The idea is that content that is shared on social networks is voluntary and accessible on demand, unlike content that was shared on traditional media whose character is imposed.

At the social media level, consumers can now choose which content they discover and enjoy. Ads can no longer stop viewers, as it is the example of YouTube ads (True View), which precede videos, consumers can now ignore these ads after five seconds. If brands or advertisers fail to attract attention in the first five seconds, they cannot complain that consumers choose to ignore the rest of their content (Kotler et al., 2017).

The use of social networks, especially via content marketing strategies, has become very useful in the marketing approach of brands, first by approaching Internet customers of their brands favorably thanks to direct and immediate contact. Then, they give brands the sense of innovation and taste of advertising, without spending too much money. Finally, social networks allow brands to benefit from word of mouth through the interconnections between their members. Brands must be very attentive and listen to advice, comments and publications left by users on the page of the brand.

Unfortunately, companies often transfer their content to social media without modifying it, considering that content is just a longer version of an advertisement. However, there is a clear difference between these two modes of communication, advertising represents the message the brand wants to convey to consumers. While content marketing disseminates the information that consumers will use to achieve their personal and professional goals (Kotler et al., 2017).

In order to ensure optimal consumer involvement, companies must take advantage of social media by creating content that, even if it does not contribute to the sale and promotion of the brand, the brand will become essential for consumers.

\section{Search Engine Optimization}

A SEO marketing logic (Search Engine Optimization) is based on the assumption that the organic traffic (free traffic, without advertising on media) of a site depends on the quality of its natural referencing, so that search engines (including, first and foremost, the unmatched Google) can locate it and especially list it correctly (Sauvage, 2016).

To do this, powerful algorithms locate the domain name (another name for the website) and analyze the content. They identify linking such as internal and external links and URL sources, and estimate the quality of each page on the topic chosen. This assumes that the website is properly structured at the base so that the bots can easily go from one page to another (Sauvage, 2016).

That is why having a content strategy is relevant from an SEO point of view: it simply gives more chances to tell Google "here's what I'm talking about, and here's my site with the most relevant pages for each subject".

The precise recipe for each of these algorithms which are more than 200, according to the Mountain View firm remains a jealously guarded secret. However, over time, SEO has been better understood by marketers, and so it has been partially debunked. 


\section{INBOUND MARKETING VS. OUTBOUND MARKETING}

Outbound marketing is interruption based marketing, perceived as the traditional model of product promotion in which people have to stop what they are doing to pay attention to the marketing message or deal with it in some other way (Rancati et al., 2015).

The difference between inbound and outbound marketing is constantly challenged. Inbound marketing has now experienced growth compared to outbound marketing which is declining. In addition, the investments in the channels of inbound marketing has come to increase significantly, compared to other channels of outbound marketing that are now either stagnant or are experiencing slow growth (Volpe, 2015).

Volpe (2015) suggests that the difference between the incoming and the outgoing is artificial and meaningless. Moreover, he proposes a cross between the strategies of the incoming and outgoing marketing, defined as a gray zone (Volpe, 2015).

"Inbound Marketing creates Top of Mind Preference, while Outbound Marketing is mainly focused on Top of Mind Awareness. With inbound content marketing programs, you can move beyond simple awareness to generate preference and implicitly brand addiction, this goal can be achieved through the positioning of a brand as the preferred choice" (Martin, 2013; Rancati et al., 2015).

Inbound Marketing's great challenge is to get the consumer to find the company online, because of the highly competitive keyword space. The timeframe in which a company is waiting for consumers to find it online might result in a sale loss (Rancati et al., 2015).

\section{CONCLUSION AND FUTURE RESEARCH}

One of the great advantages of the internet is that it reaches consumers around the world, the globalization of business promises a lot thanks to the advent of new technologies.

The inbound marketing is a strategy that aims to attract prospects to a company's website, convert them to leads and then further, to lifelong customers and brand promoters.

In this article, we have proposed a comparison between Inbound and Outbound Marketing strategies. That said, based on this paper's propositions, we conclude that both Inbound and Outbound strategies are complementary for the success of any viable marketing campaign. Both strategies have their pros and they can both be used together, often to a greater effect, creating a synergy.

Indeed, the mix of outbound and inbound marketing strategies can work most effectively and reach the most amount of people when done hand-in-hand

Nevertheless, Inbound Marketing is primarily content marketing and its efficiency is superior to the Outbound Marketing efficiency because inbound aims to identify the target customers and adapt the communication, however it allows a better targeting of the audience and a better adaptation of the company's communication mechanisms.

\section{Future Research}

This theoretical article can be extended to encompass an empirical study that calculates through different metrics the impact of inbound marketing practices on consumers, via data gathered from websites such as www.Alexa.com. 


\section{REFERENCES}

Cantin (2014). Inbound marketing definition. Retrieved May 5, 2018, from https://www.mychefcom.com/blog/definition-de-linbound-marketing

Chaffey, D., Mayer, R., Johnston, K., \& Ellis-Chadwick, F. (2000). Internet marketing. Edinburgh: Pearson Education.

Dabi-Schwebel, G. (2017). L'inbound marketing permet de convertir son audience en clients Retrieved May 5, 2018, from http://www.entreprendre.fr/gabriel-dabi-schwebel

Godin, S. (1999). Permission marketing. NY, USA: Simon \& Schuster.

Goodwin, T. (2013). Inbound marketing vs. outbound marketing: What's the difference? Retrieved May 4, 2018, from http://boldthinkcreative.com/inbound-marketing-vs-outbound-marketing-whats-thedifference/

Gunelius, S. (2011). Content Marketing for Dummies. NY, USA: Wiley.

Halligan, B., \& Shah, D. (2014). Inbound marketing - Attract, Engage and delight customers online. New Jersey, USA: Wiley.

Halligan, B., Shah, D., \& Scott, D.M. (2009). Inbound Marketing: Get Found Using Google, Social Media, and Blogs. New Jersey, USA :Wiley.

Handley, A., \& Chapman, C.C. (2010). Content Rules. NY, USA: Wiley.

Hubspot. (2016). The Inbound Methodology - The best way to turn strangers into customers and promoters of your business. Retrieved May 5, 2018, from http://www.hubspot.com/inboundmarketing>

InboundValue.com. (2018). What is Inbound Marketing? Retrieved May 5, 2018, from https://www.inboundvalue.com/definition-inbound-marketing/

Keyes, J. (2006). Knowledge management, business intelligence and content management. NY, USA: Auerbach Publications.

Kotler, P., Kartajaya, H., \& Setiawan, I. (2017). Marketing 4.0: Moving from Traditional to Digital. NY, USA: Wiley.

Ladd, T., \& Kendall, L. (2017). Resolving the Risk Paradox: Entrepreneurial Cognition in the Lean Startup Method. Journal of Applied Business and Economics, 19(11). Retrieved from https://articlegateway.com/index.php/JABE/article/view/775

Marketing-Schools.org. (2016). Inbound Marketing - Explore the Strategy of Inbound Marketing. Retrieved May 5, 2018, from http://www.marketing-schools.org/typesof-Marketing/inboundmarketing.html

Martin, T. (2013). Inbound Marketing versus Outbound Marketing Compared. Retrieved November 11, 2018, from http://www.conversedigital.com/social-selling-posts/inbound-marketing-versusoutbound-marketing-salesprospecting

Patrutiu-Baltes, L. (2016). Inbound Marketing - The most important digital marketing strategy. Bulletin of the Transilvania University of Braşov, Series V: Economic Sciences, 9(58), 2, 61-68.

Pulizzi, J. (2012). The Rise of Storytelling as the New Marketing. Publishing Research Quarterly, 28(2), $116-123$.

Pulizzi, J., \& Barrett, N. (2009). Get content. Get customers. NY, USA: McGraw Hill.

Pullizi, J. (2011). The 7 Business Goals of Content Marketing: Inbound Marketing Isn't Enough. Retrieved May 5, 2018, from https://contentmarketinginstitute.com/2011/11/content-marketinginbound-marketing/

Ramos, R. (2014). Content Marketing: Insider's secret to online sales and lead generation. NY, USA: One Night Expert Publishing.

Rancati, E., Codignola, F., \& Capatina, A. (2015). Inbound and Outbound Marketing Techniques: A Comparison Between Italian and Romanian Pure Players and Click and Mortar Companies. In International Conference "Risk in Contemporary Economy" (Xvith Edition). Galati, Romania.

Rouse, M. (2014). What is inbound marketing. Retrieved May 5, 2018, from https://www.techtarget.com/contributor/Margaret-Rouse 
Sauvage, M. (2016). Pourquoi SEO et inbound marketing vont-ils bien ensemble? Retrieved November 11, 2018, from https://www.inboundvalue.com/blog/pourquoi-seo-vs-inbound-marketing

Slater, D. (2014). Content marketing: Recycling and reuse. New York: i30 Media Corporation.

Venisse, M. (2016). Créer sa stratégie inbound marketing globale. Retrieved May 4, 2018, from https://www.1min30.com/formations-e-learning/strategie-inbound-marketing-1287455005

Volpe, M. (2015). Inbound marketing versus outbound marketing: A pointless debate. Retrieved November 11, 2018, from https://www.i-scoop.eu/inbound-marketing-versus-outboundmarketing-pointless-debate/

Wainwright, C. (2014). Confessions of a Content Marketer. Retrieved November 11, 2018, from https://blog.hubspot.com/blog/tabid/6307/bid/28681/confessions-of-a-content-marketer.aspx>

\section{APPENDIX}

TABLE 1

INBOUND AND OUTBOUND MARKETING COMPARISON

\begin{tabular}{|c|c|c|}
\hline Outbound Marketing & Inbound Marketing & Grey Zone \\
\hline $\begin{array}{ll}\text { - } & \text { Print Ads } \\
\text { - } & \text { Direct } \\
& \text { Mailings } \\
\text { - } & \text { Outbound } \\
& \text { Call Center } \\
\text { - } & \text { TV, Radio } \\
& \text { advertising } \\
\text { - } & \text { Intrusive } \\
& \text { online ads }\end{array}$ & $\begin{array}{ll}\text { - } & \text { Content } \\
& \text { Marketing } \\
\text { - } & \text { Blog Marketing } \\
\text { - } & \text { SEO } \\
\text { - } & \text { Social Media } \\
& \text { Marketing } \\
\text { - } & \text { Viral } \\
& \text { Marketing } \\
\text { - } & \text { Podcasts } \\
\text { - } & \text { RSS }\end{array}$ & $\begin{array}{l}\text { - } \quad \text { E-mail Marketing } \\
\text { - } \text { Display Advertising } \\
\text { - Search Engine Ads } \\
\text { - Social media (when used as } \\
\text { broadcasting medium) }\end{array}$ \\
\hline
\end{tabular}

Source: adapted from Volpe (2015) and Rancati et al. (2015)

\section{TRANSLATED VERSION: SPANISH}

Below is a rough translation of the insights presented above. This was done to give a general understanding of the ideas presented in the paper. Please excuse any grammatical mistakes and do not hold the original authors responsible for these mistakes.

\section{VERSION TRADUCIDA: ESPAÑOL}

A continuación se muestra una traducción aproximada de las ideas presentadas anteriormente. Esto se hizo para dar una comprensión general de las ideas presentadas en el documento. Por favor, disculpe cualquier error gramatical y no responsabilite a los autores originales de estos errores.

\section{INTRODUCCIÓN}

El desarrollo de la estrategia de marketing ha experimentado una valiosa evolución, con la existencia de la comunicación digital y las redes sociales, la creación de contenidos ha cambiado. Hace varios años, las empresas utilizaban la estrategia tradicional, como enviar un mensaje a una audiencia, con el fin de empujarla hacia su empresa o producto, en contra del desarrollo de las nuevas estrategias de marketing, a 
saber, el Inbound Marketing, una nueva forma de atraer a la audiencia hacia la empresa, mediante la creación de contenidos más innovadores.

El estado del Inbound Marketing (Hubspot, 2013) describe el Outbound Marketing o marketing tradicional como una técnica utilizada por las empresas con el fin de atraer a un gran público, con la esperanza de obtener una retroalimentación. Utilizando la estrategia outbound, basada principalmente en la difusión del mensaje y la publicidad, sin estudiar el target, con el fin de recibir una respuesta efectiva y generar más ventas e ingresos.

El outbound marketing es el marketing basado en interrupciones, percibido como el modelo tradicional de promoción de productos en el que las personas tienen que dejar de hacer lo que están haciendo para prestar atención al mensaje de marketing o tratarlo de alguna otra manera (Rancati et al., 2015). El outbound marketing es también una estrategia en la que una empresa anuncia sus productos y servicios presentando información a los consumidores, incluso si no están buscando esos productos o servicios (Goodwin, 2013). Sin embargo, esta estrategia ya no es efectiva para transmitir el mensaje y hacer tráfico en el mundo web, es por eso que las empresas, hoy en día, tienden a explorar nuevas estrategias como el enfoque de Inbound Marketing para obtener más resultados de Internet.

En la primera segunda sección de este artículo, definiremos el inbound marketing y obtendremos una visión general de la aparición de este concepto. En la tercera sección, explicaremos los componentes del inbound marketing. En cuarto apartado, compararemos el Outbound y el Inbound Marketing para saber si representan estrategias independientes o complementarias.

\section{DEFINICIONES DE INBOUND MARKETING}

El inbound marketing es la estrategia de conectar con clientes potenciales a través de materiales y experiencias que encuentran útiles. Utilizando medios como blogs y redes sociales, los especialistas en marketing esperan entretener e informar a los espectadores con el contenido que buscan por sí mismos (Marketing-Schools.org, 2016).

Por otro lado, el inbound marketing puede explicarse como una forma de marketing que requiere una orientación muy cuidadosa de la audiencia y una comunicación personalizada a través de contenido de alta calidad (Patrutiu-Baltes, 2016).

Además, Venisse (2016) definió el inbound marketing como una forma de convertirse en sus propios medios para que la audiencia se convierta más fácilmente en clientes. La implementación de la estrategia global de inbound marketing requiere el respeto de ciertos pasos esenciales (Venisse, 2016). El primer paso es desarrollar sus propios medios y construir una audiencia calificada. Para una empresa que establece esta estrategia, es importante ofrecer contenido interesante en su sitio, su blog o su página de red social, para atraer y sostener una audiencia.

Además, Schwebel (2017) sugiere que el inbound marketing tiene el potencial de desarrollar una marca para convertirse en su propio medio, es decir, ofrecer contenido que sea lo suficientemente inteligente y relevante como para construir una audiencia, en lugar de anuncios intrusivos (Dabi-Schwebel, 2017).

Cantin (2014) define que el inbound marketing es una estrategia de marketing digital dirigida a acercar al cliente a la empresa en lugar de recogerlo. El prospecto se siente atraído por contenido relevante posicionado de acuerdo a fases de madurez de compras y niveles de compromiso. Por lo tanto, en esta línea de pensamiento, también podríamos hablar sobre el marketing de adquisición (Cantin, 2014).

Definimos el inbound marketing también como una estrategia de marketing digital cuya idea general es atraer continuamente prospectos potenciales con contenido de calidad. Para ello, el Inbound Marketing utiliza una metodología de cuatro pasos: Atraer, Convertir, Transformar y Retener (InboundValue.com, 2018).

Además, como propuso Rouse (2014), el inbound marketing se puede definir "como una técnica que se centra en acercar a los consumidores o leads a través de contenido relevante, para que los consumidores busquen la empresa en lugar de lo contrario. El concepto de inbound marketing facilita las tareas a los consumidores que desean bienes, productos o servicios relevantes" (Rouse, 2014). 
Hubspot (2018) define el Inbound Marketing como una estrategia que atrae a los clientes con contenido relevante y útil y agrega valor en cada paso del viaje de compra del cliente. Con el inbound marketing, los clientes potenciales encuentran su camino a través de canales como blogs, motores de búsqueda y redes sociales (Hubspot, 2016). A diferencia del Outbound Marketing, el Inbound Marketing no necesita luchar por la atención de los clientes potenciales. Al crear contenido que aborda los problemas y necesidades de los clientes ideales, la marca atrae a prospectos calificados y genera confianza y credibilidad. Por lo tanto, el inbound marketing es un enfoque sistémico que permite que cada marca se convierta en sus propios medios, crear una audiencia y convertirla en prospectos y luego en clientes. Todo un proceso funciona bien en los detalles al tiempo que elimina cualquier falla (Dabi-Schwebel, 2017).

\section{APARICIÓN DEL INBOUND MARKETING}

El término «Inbound» fue utilizado por Brian Halligan (Halligan et al., 2009). El término inbound se refiere a atraer y convertir a las personas en clientes duraderos.

Correspondiente a los creadores de inbound marketing Brian Halligan y Dhamesh Shah, las necesidades de este concepto innovador se crearon a partir del hecho de que las personas están enfermas y cansadas de ser interrumpidas con mensajes tradicionales de outbound marketing. Además, las tácticas de marketing utilizadas con éxito en el pasado ya no producía los resultados del deseo (Halligan et al., 2009).

Todo comenzó cuando Shah comenzó un blog sobre startups y emprendimiento, luego, notó que su blog ganó un tráfico masivo más que los sitios web propiedad de empresas con equipos de marketing profesionales y grandes presupuestos. Después de muchos análisis y discusiones, salieron con una simple observación de que "las personas no querían ser interrumpidas por los vendedores o acosadas por los vendedores, quieren ser ayudadas" (Halligan y Shah, 2014).

La idea principal del inbound marketing es utilizar técnicas de marketing que intentarán captar el interés de los clientes, compartiendo contenido creativo y útil que se comparte a través de múltiples canales en línea, incluidos los motores de búsqueda y las redes sociales.

Incluso si el inbound marketing fue introducido por primera vez en 2005 por Halligan y Shah, en realidad no es un concepto nuevo, es similar al método de marketing llamado "Marketing de contenidos" y también se puede asociar al "Marketing narrative", es decir, una técnica utilizada mucho antes de la aparición de Internet (Pulizzi, 2012).

Además, el marketing de contenidos se define como un proceso de marketing que tiene como objetivo desarrollar contenido relevante con el fin de atraer e involucrar a un público objetivo, obteniendo así una acción rentable del consumidor (Pulizzi, 2012).

Además, el marketing de contenidos debe incluir la planificación estratégica, la creación de contenido y la distribución para múltiples etapas del ciclo de compra a múltiples clientes, lo que significa que una estrategia completa de marketing de contenidos debe incorporar el principio de Inbound marketing (Pulizzi, 2011).

Una de las técnicas del inbound marketing conocida como la optimización de motores de búsqueda (SEO) se practicó durante mucho tiempo antes del concepto de marketing de contenidos, y el SEO se utiliza principalmente para aumentar el número de visitantes que provienen de los motores de búsqueda.

Las palabras clave fueron consideradas el elemento más importante para lograr un alto ranking, luego, con el tiempo y después de la introducción de muchas actualizaciones que se incluyeron, como Panda en 2011, Penguin en 2012 y la actualización de Hummingbird en 2013 (Wainwright, 2014), esta importancia de las palabras clave disminuyó. Además, debido a estas actualizaciones, el SEO se convierte en una tarea muy difícil. A partir de aquí, el inbound marketing entra en su lugar, según Rand Fishkin, el SEO es una táctica, y el inbound marketing es una estrategia.

El email marketing también se refiere a uno de los elementos importantes del inbound marketing basado en el envío de newsletter, artículos u otros, a través de un correo electrónico. Es un tipo de marketing basado en el permiso dado por los visitantes, también se conoce como marketing de permiso, es decir, un concepto que fue desarrollado por Seth Godin (Godin, 1999). 


\section{COMPONENTES DEL INBOUND MARKETING}

\section{Marketing de Contenidos}

Aunque el marketing de contenidos se ha mantenido a la sombra del marketing tradicional dada la falta de tecnología en ese momento, ha logrado hoy en día establecerse como una estrategia efectiva especialmente con el auge de las nuevas tecnologías.

Chaffey y Al (2000) definen el contenido como un diseño de texto e información gráfica que forma una página web. Un buen contenido es una piedra angular para atraer clientes a un sitio web y retener su interés mientras provoca visitas repetidas. El marketing de contenidos también puede ser el contenido de la intranet de la empresa o el contenido de Internet de la empresa, incluidas las bases de datos y varios tipos de conocimiento (Chaffey y Al, 2000).

Por su parte, Pulizzi y Barret (2009) también definen el marketing de contenidos como una técnica de marketing que consiste en crear, publicar y distribuir contenido relevante, útil y valioso para obtener beneficios de los consumidores. El marketing de contenidos tiende a tener medios en lugar de alquilar. Se define como un proceso de marketing que atrae y retiene a los clientes mediante la creación constante de contenido que tiene como objetivo cambiar o evolucionar el comportamiento del consumidor (Pulizzi, J. y Barrett, 2009).

Handley y Chapman (2010) argumentan que el marketing de contenidos es todo lo que un individuo u organización crea o comparte para contar sus historias. El contenido es conversacional, humano y no busca constantemente vender. Debe ser un estado mental tímido y alentado (Handley y Chapman, 2010).

Gunelius (2011) define el marketing de contenidos como un proceso de promoción directa e indirecta de una empresa o marca a través del beneficio de un contenido de texto, video o audio a la vez, en entornos online y offline. Puede aparecer en formato largo (blogs, artículos, libros electrónicos y sonido), en formato corto (Twitter, Facebook, imágenes ...), o en forma convencional, (compartir contenido a través de Twitter o participar en una discusión activa a través de comentarios en publicaciones de blog o a través de foros en línea) (Gunelius, 2011).

Staler (2014) explica que el marketing de contenidos es una técnica dinámica dirigida a atraer clientes, es un proceso de compartir experiencia y conocimiento con el fin de atraer clientes potenciales y establecer una relación con ellos (Slater, 2014).

Ramos (2014) define el marketing de contenidos como una técnica de marketing de alta calidad, contenido relevante que pretende ser educativo y movilizador para adquirir el público objetivo (Ramos, 2014).

Kotler et al. (2017) definen el marketing de contenidos como un enfoque de marketing que consiste en crear, organizar, distribuir y amplificar contenido interesante, relevante y útil para un grupo de audiencia claramente definido para crear conversaciones de contenido. El marketing de contenidos también es otra forma de periodismo de marca y de marca que crea vínculos más estrechos entre las marcas y los clientes. En última instancia, el marketing de contenidos se trata de planificar, crear, promover y monitorear el rendimiento para un público objetivo, siempre con el objetivo de la satisfacción del cliente y la generación de ingresos en mente (Kotler et al., 2017).

\section{Marketing en Redes Sociales}

Las características de las redes sociales y su inmensa popularidad han revolucionado prácticas comerciales como la publicidad y la promoción.

Las redes sociales son espacios originales. Pueden dar lugar a compañeros de comunicación convencionales de arriba hacia abajo (uno a muchos), pero también cambiar esta comunicación vertical y unilateral hacia un diálogo entre el usuario y la marca (uno a uno) o incluso más entre los propios internautas mismos (muchos a muchos).

Sin embargo, los consumidores están cada vez menos interesados en la publicidad tradicional, lo que ejerce una enorme presión sobre los vendedores. El desafío radica en difundir información compleja a través de mensajes sin abrumar a los consumidores. La actitud de los consumidores de hoy ha cambiado profundamente, el tiempo de apreciación y lectura del mensaje ha disminuido mucho. La capacidad de 
atención promedio de los humanos ha disminuido de 12 segundos en 2000 a 8 segundos en 2013. Aún más, esta atención está destinada a disminuir en el futuro, debido al volumen masivo y abrumador de mensajes que bombardean constantemente los dispositivos móviles conectados que requieren atención inmediata del consumidor (Kotler et al., 2017).

Las redes sociales han jugado un papel muy importante en estos cambios. En el pasado, los consumidores se limitaban a los comerciales generados en los medios tradicionales porque no tenían muchas opciones. Hoy en día, con la llegada de las redes sociales, los consumidores tienen una gran cantidad de contenido generado por empresas o incluso por otros consumidores que encuentran más creíble e interesante. La idea es que el contenido que se comparte en las redes sociales sea voluntario y accesible bajo demanda, a diferencia del contenido que se compartió en los medios tradicionales cuyo carácter se impone.

A nivel de redes sociales, los consumidores ahora pueden elegir qué contenido descubren y disfrutan. Los anuncios ya no pueden detener a los espectadores, como es el ejemplo de los anuncios de YouTube (True View), que preceden a los videos, los consumidores ahora pueden ignorar estos anuncios después de cinco segundos. Si las marcas o los anunciantes no logran llamar la atención en los primeros cinco segundos, no pueden quejarse de que los consumidores elijan ignorar el resto de su contenido (Kotler et al., 2017).

El uso de las redes sociales, especialmente a través de estrategias de marketing de contenidos, se ha vuelto muy útil en el enfoque de marketing de las marcas, primero al acercarse favorablemente a los clientes de Internet de sus marcas gracias al contacto directo e inmediato. Luego, le dan a las marcas el sentido de la innovación y el gusto de la publicidad, sin gastar demasiado dinero. Finalmente, las redes sociales permiten a las marcas beneficiarse del boca a boca a través de las interconexiones entre sus miembros. Las marcas deben estar muy atentas y escuchar consejos, comentarios y publicaciones que dejan los usuarios en la página de la marca.

Desafortunadamente, las empresas a menudo transfieren su contenido a las redes sociales sin modificarlo, teniendo en cuenta que el contenido es solo una versión más larga de un anuncio. Sin embargo, existe una clara diferencia entre estos dos modos de comunicación, la publicidad representa el mensaje que la marca quiere transmitir a los consumidores. Mientras que el marketing de contenidos difunde la información que los consumidores utilizarán para lograr sus objetivos personales y profesionales (Kotler et al., 2017).

Para garantizar una óptima implicación del consumidor, las empresas deben aprovechar las redes sociales creando contenidos que, aunque no contribuyan a la venta y promoción de la marca, la marca se convertirá en imprescindible para los consumidores.

\section{Optimización de Motores de Búsqueda}

Una lógica de marketing SEO (Search Engine Optimization) se basa en la suposición de que el tráfico orgánico (tráfico libre, sin publicidad en los medios) de un sitio depende de la calidad de su referencia natural, de modo que los motores de búsqueda (incluido, ante todo, el inigualable Google) puedan localizarlo y especialmente listarlo correctamente (Sauvage, 2016).

Para hacer esto, poderosos algoritmos localizan el nombre de dominio (otro nombre para el sitio web) y analizan el contenido. Identifican enlaces como enlaces internos y externos y fuentes de URL, y estiman la calidad de cada página sobre el tema elegido. Esto supone que el sitio web está correctamente estructurado en la base para que los bots puedan ir fácilmente de una página a otra (Sauvage, 2016).

Es por eso que tener una estrategia de contenido es relevante desde el punto de vista SEO: simplemente da más oportunidades de decirle a Google "esto es de lo que estoy hablando, y aquí está mi sitio con las páginas más relevantes para cada tema".

La receta precisa para cada uno de estos algoritmos que son más de 200, según la firma de Mountain View, sigue siendo un secreto celosamente guardado. Sin embargo, con el tiempo, el SEO ha sido mejor entendido por los especialistas en marketing, por lo que ha sido parcialmente desacreditado. 


\section{INBOUND MARKETING VS. MARKETING SALIENTE}

El outbound marketing es el marketing basado en la interrupción, percibido como el modelo tradicional de promoción de productos en el que las personas tienen que dejar de hacer lo que están haciendo para prestar atención al mensaje de marketing o tratarlo de alguna otra manera (Rancati et al., 2015).

La diferencia entre el inbound y el outbound marketing se cuestiona constantemente. El inbound marketing ahora ha experimentado un crecimiento en comparación con el outbound marketing, que está disminuyendo. Además, las inversiones en los canales de inbound marketing han llegado a aumentar significativamente, en comparación con otros canales de outbound marketing que ahora están estancados o están experimentando un crecimiento lento (Volpe, 2015).

Volpe (2015) sugiere que la diferencia entre lo entrante y lo saliente es artificial y sin sentido. Además, propone un cruce entre las estrategias del marketing entrante y saliente, definido como una zona gris (Volpe, 2015).

"El Inbound Marketing crea Top of Mind Preference, mientras que Outbound Marketing se centra principalmente en Top of Mind Awareness. Con los programas de inbound content marketing, se puede ir más allá de la simple conciencia para generar preferencia e implícitamente adicción a la marca, este objetivo se puede lograr a través del posicionamiento de una marca como la opción preferida" (Martin, 2013; Rancati et al., 2015).

El gran reto del Inbound Marketing es conseguir que el consumidor encuentre la empresa online, debido al espacio de palabras clave altamente competitivo. El período de tiempo en el que una empresa está esperando que los consumidores lo encuentren en línea podría resultar en una pérdida de venta (Rancati et al., 2015).

\section{CONCLUSIÓN E INVESTIGACIÓN FUTURA}

Una de las grandes ventajas de internet es que llega a consumidores de todo el mundo, la globalización de los negocios promete mucho gracias a la llegada de las nuevas tecnologías.

El inbound marketing es una estrategia que tiene como objetivo atraer prospectos al sitio web de una empresa, convertirlos en clientes potenciales y luego, a clientes de por vida y promotores de marca.

En este artículo, hemos propuesto una comparación entre las estrategias de Inbound y Outbound Marketing. Dicho esto, basándonos en las propuestas de este documento, concluimos que tanto las estrategias Inbound como Outbound son complementarias para el éxito de cualquier campaña de marketing viable. Ambas estrategias tienen sus ventajas y ambas se pueden usar juntas, a menudo con un mayor efecto, creando una sinergia.

De hecho, la combinación de estrategias de marketing saliente e inbound puede funcionar de manera más efectiva y llegar a la mayor cantidad de personas cuando se hace de la mano.

Sin embargo, el Inbound Marketing es principalmente marketing de contenidos y su eficiencia es superior a la eficiencia del Outbound Marketing porque el inbound tiene como objetivo identificar a los clientes objetivo y adaptar la comunicación, sin embargo permite una mejor orientación de la audiencia y una mejor adaptación de los mecanismos de comunicación de la empresa.

\section{Investigación Futura}

Este artículo teórico se puede ampliar para abarcar un estudio empírico que calcula a través de diferentes métricas el impacto de las prácticas de inbound marketing en los consumidores, a través de datos recopilados de sitios web como www.Alexa.com.

\section{TRANSLATED VERSION: FRENCH}

Below is a rough translation of the insights presented above. This was done to give a general understanding of the ideas presented in the paper. Please excuse any grammatical mistakes and do not hold the original authors responsible for these mistakes. 


\section{VERSION TRADUITE: FRANÇAIS}

Voici une traduction approximative des idées présentées ci-dessus. Cela a été fait pour donner une compréhension générale des idées présentées dans le document. Veuillez excuser toutes les erreurs grammaticales et ne pas tenir les auteurs originaux responsables de ces erreurs.

\section{INTRODUCTION}

Le développement de la stratégie marketing a subi une évolution précieuse, avec l'existence de la communication numérique et des réseaux sociaux, la création de contenu a été modifiée. Il y a plusieurs années, les entreprises utilisaient la stratégie traditionnelle, comme envoyer un message à un public, afin de le pousser vers son entreprise ou son produit, à l'opposé du développement des nouvelles stratégies marketing, à savoir l'Inbound Marketing, une nouvelle façon d'attirer le public vers l'entreprise, en créant un contenu plus innovant.

The State of Inbound Marketing (Hubspot, 2013) décrit l'Outbound Marketing ou marketing traditionnel comme une technique utilisée par les entreprises afin d'attirer un large public, dans l'espoir d'obtenir un retour d'information. Utiliser la stratégie outbound, principalement basée sur la diffusion du message et de la publicité, sans étudier la cible, afin de recevoir une réponse efficace et de générer plus de ventes et de revenus.

Le marketing sortant est un marketing basé sur l'interruption, perçu comme le modèle traditionnel de promotion de produits dans lequel les gens doivent arrêter ce qu'ils font pour prêter attention au message marketing ou le traiter d'une autre manière (Rancati et al., 2015). Le marketing sortant est également une stratégie dans laquelle une entreprise fait de la publicité pour ses produits et services en présentant des informations aux consommateurs même s'ils ne recherchent pas ces produits ou services (Goodwin, 2013). Cependant, cette stratégie n'est plus efficace pour faire passer le message et faire du trafic dans le monde du Web, c'est pourquoi les entreprises, de nos jours, ont tendance à explorer de nouvelles stratégies telles que l'approche Inbound Marketing afin d'obtenir plus de résultats d'Internet.

Dans la première deuxième section de cet article, nous définirons l'inbound marketing et aurons un aperçu de l'émergence de ce concept. Dans la troisième section, nous expliquerons les composants de l'inbound marketing. Dans la quatrième section, nous comparerons l'Outbound et l'Inbound Marketing afin de savoir s'ils représentent des stratégies indépendantes ou complémentaires.

\section{DÉFINITIONS DE L'INBOUND MARKETING}

L'inbound marketing est la stratégie de connexion avec les clients potentiels à travers des matériaux et des expériences qu'ils trouvent utiles. En utilisant des médias comme les blogs et les réseaux sociaux, les spécialistes du marketing espèrent divertir et informer les téléspectateurs avec le contenu qu'ils recherchent par eux-mêmes (Marketing-Schools.org, 2016).

D'autre part, l'inbound marketing peut s'expliquer comme une forme de marketing qui nécessite un ciblage très minutieux de l'audience et une communication personnalisée via un contenu de haute qualité (Patrutiu-Baltes, 2016).

De plus, Venisse (2016) a défini l'inbound marketing comme un moyen de devenir son propre média afin que le public se convertisse plus facilement en clients. La mise en œuvre de la stratégie globale d'inbound marketing nécessite le respect de certaines étapes essentielles (Venisse, 2016). La première étape consiste à développer ses propres médias et à construire un public qualifié. Pour une entreprise qui met en place cette stratégie, il est important de proposer des contenus intéressants sur son site, son blog ou sa page de réseau social, afin d'attirer et de pérenniser un public.

En outre, Schwebel (2017) suggère que l'inbound marketing a le potentiel de développer une marque pour devenir son propre média, c'est-à-dire d'offrir un contenu suffisamment intelligent et pertinent pour construire un public, au lieu de publicités intrusives (Dabi-Schwebel, 2017). 
Cantin (2014) définit l'inbound marketing comme une stratégie de marketing numérique visant à amener le client dans l'entreprise plutôt que de le récupérer. Le prospect est attiré par un contenu pertinent positionné en fonction des phases de maturité des achats et des niveaux d'engagement. Par conséquent, dans cette ligne de pensée, nous pourrions également parler du marketing d'acquisition (Cantin, 2014).

Nous définissons également l'inbound marketing comme une stratégie de marketing numérique dont l'idée générale est d'attirer en permanence des prospects potentiels avec un contenu de qualité. Pour ce faire, l'Inbound Marketing utilise une méthodologie en quatre étapes : Attirer, Convertir, Transformer et Conserver (InboundValue.com, 2018).

De plus, comme Rouse (2014) l'a proposé, l'inbound marketing peut être défini « comme une technique qui se concentre sur l'apport de consommateurs ou de prospects via un contenu pertinent, de sorte que les consommateurs recherchent l'entreprise au lieu du contraire. Le concept d'inbound marketing facilite les tâches des consommateurs qui veulent des biens, des produits ou des services pertinents » (Rouse, 2014).

Hubspot (2018) définit l'Inbound Marketing comme une stratégie qui attire les clients avec un contenu pertinent et utile et ajoute de la valeur à chaque étape du parcours d'achat client. Avec l'inbound marketing, les clients potentiels trouvent leur chemin à travers des canaux tels que les blogs, les moteurs de recherche et les médias sociaux (Hubspot, 2016). Contrairement à l'Outbound Marketing, l'Inbound Marketing n'a pas besoin de se battre pour attirer l'attention des clients potentiels. En créant un contenu qui répond aux problèmes et aux besoins des clients idéaux, la marque attire des prospects qualifiés et renforce la confiance et la crédibilité. Par conséquent, l'inbound marketing est une approche systémique qui permet à chaque marque de devenir son propre média, de créer un public et de le convertir en prospects puis en clients. Tout un processus fonctionne bien sur les détails tout en éliminant toute défaillance (Dabi-Schwebel, 2017).

\section{ÉMERGENCE DE L'INBOUND MARKETING}

Le terme « Inbound » a été utilisé par Brian Halligan (Halligan et al., 2009). Le terme inbound fait référence à l'attraction et à la conversion de personnes en clients durables.

Correspondant aux créateurs de l'inbound marketing Brian Halligan et Dhamesh Shah, les besoins de ce concept innovant ont été créés à partir du fait que les gens en ont marre d'être interrompus par des messages traditionnels de marketing sortant. De plus, les tactiques de marketing utilisées avec succès dans le passé ne produisaient plus les résultats désirés (Halligan et al., 2009).

Tout a commencé lorsque Shah a lancé un blog sur les startups et l'entrepreneuriat, puis, il a remarqué que son blog gagnait un trafic massif plus que les sites Web appartenant à des entreprises avec des équipes de marketing professionnelles et de gros budgets. Après beaucoup d'analyses et de discussions, ils sont sortis avec une simple observation que « les gens ne voulaient pas être interrompus par les spécialistes du marketing ou harcelés par les vendeurs, ils voulaient être aidés » (Halligan et Shah, 2014).

L'idée principale de l'inbound marketing est d'utiliser des techniques de marketing qui tenteront de capter l'intérêt des clients, en partageant du contenu créatif et utile qui est partagé via plusieurs canaux en ligne, y compris les moteurs de recherche et les médias sociaux.

Même si l'inbound marketing a été introduit pour la première fois en 2005 par Halligan et Shah, il ne s'agit en fait pas d'un concept nouveau, il est similaire à la méthode de marketing appelée "Content marketing » et il peut également être associé au « Storytelling marketing », à savoir une technique utilisée bien avant l'émergence d'Internet (Pulizzi, 2012).

De plus, le marketing de contenu est défini comme un processus de marketing qui vise à développer un contenu pertinent afin d'attirer et d'engager un public cible, obtenant ainsi une action rentable du consommateur (Pulizzi, 2012).

En outre, le marketing de contenu doit inclure la planification stratégique, la création et la distribution de contenu pour plusieurs étapes du cycle d'achat à plusieurs clients, ce qui signifie qu'une stratégie complète de marketing de contenu doit intégrer le principe de l'Inbound marketing (Pulizzi, 2011).

L'une des techniques de l'inbound marketing connue sous le nom d'optimisation des moteurs de recherche (SEO) a été pratiquée pendant longtemps avant le concept de marketing de contenu, et le 
référencement est principalement utilisé pour augmenter le nombre de visiteurs provenant des moteurs de recherche.

Les mots-clés ont été considérés comme l'élément le plus important pour atteindre un classement élevé, puis, avec le temps et après l'introduction de nombreuses mises à jour incluses, telles que Panda en 2011, Penguin en 2012 et la mise à jour Hummingbird en 2013 (Wainwright, 2014), cette importance des motsclés a diminué. De plus, en raison de ces mises à jour, le référencement devient une tâche très difficile. À partir de là, l'inbound marketing se mettent en place, selon Rand Fishkin, le référencement est une tactique et l'inbound marketing est une stratégie.

Le marketing par e-mail fait également référence à l'un des éléments importants de l'inbound marketing basé sur l'envoi de newsletters, d'articles ou autres, via un e-mail. C'est un type de marketing basé sur la permission donnée par les visiteurs, est-il également connu sous le nom de marketing d'autorisation, à savoir un concept qui a été développé par Seth Godin (Godin, 1999).

\section{COMPOSANTES DE L'INBOUND MARKETING}

\section{Marketing de Contenu}

Bien que le marketing de contenu soit resté dans l'ombre du marketing traditionnel compte tenu du manque de technologie à cette époque, il a réussi aujourd'hui à s'imposer comme une stratégie efficace, en particulier avec l'essor des nouvelles technologies.

Chaffey et Al (2000) définissent le contenu comme une conception textuelle et graphique de l'information qui forme une page Web. Un bon contenu est une clé de voûte pour attirer les clients sur un site Web et conserver leur intérêt tout en provoquant des visites répétées. Le marketing de contenu peut également être le contenu intranet de l'entreprise ou le contenu Internet de l'entreprise, y compris les bases de données et divers types de connaissances (Chaffey et Al, 2000).

Pour leur part, Pulizzi et Barret (2009) définissent également le marketing de contenu comme une technique de marketing qui consiste à créer, publier et distribuer du contenu pertinent, utile et précieux pour obtenir des profits des consommateurs. Le marketing de contenu a tendance à avoir des médias plutôt que de les louer. Il est défini comme un processus de marketing qui attire et fidélise les clients en créant constamment du contenu qui vise à changer ou à faire évoluer le comportement des consommateurs (Pulizzi, J. et Barrett, 2009).

Handley et Chapman (2010) soutiennent que le marketing de contenu est tout ce qu'un individu ou une organisation crée ou partage pour raconter ses histoires. Le contenu est conversationnel, humain et ne cherche pas constamment à vendre. Ce doit être un état d'esprit à la fois timide et encouragé (Handley et Chapman, 2010).

Gunelius (2011) définit le marketing de contenu comme un processus de promotion directe et indirecte d'une entreprise ou d'une marque grâce au bénéfice d'un contenu texte, vidéo ou audio à la fois, dans des environnements en ligne et hors ligne. Il peut apparaître sous forme longue (blogs, articles, e-books et son), sous forme courte (Twitter, Facebook, images...), ou sous forme conventionnelle, (partage de contenu via Twitter ou participation à une discussion active via des commentaires sur des articles de blog ou via des forums en ligne) (Gunelius, 2011).

Staler (2014) explique que le marketing de contenu est une technique dynamique visant à attirer des clients, c'est un processus de partage d'expertise et de connaissances afin d'attirer des clients potentiels et d'établir une relation avec eux (Slater, 2014).

Ramos (2014) définit le marketing de contenu comme une technique de marketing de haute qualité, un contenu pertinent qui vise à être éducatif et mobilisateur pour acquérir le public cible (Ramos, 2014).

Kotler et al. (2017) définissent le marketing de contenu comme une approche marketing qui consiste à créer, organiser, distribuer et amplifier du contenu intéressant, pertinent et utile pour un groupe d'audience clairement défini afin de créer des conversations de contenu. Le marketing de contenu est également une autre forme de branding et de journalisme de marque qui crée des liens plus étroits entre les marques et les clients. En fin de compte, le marketing de contenu consiste à planifier, créer, promouvoir et surveiller les 
performances d'un public ciblé, toujours dans le but de satisfaire la clientèle et de générer des revenus (Kotler et al., 2017).

\section{Marketing des Médias Sociaux}

Les caractéristiques des médias sociaux et leur immense popularité ont révolutionné les pratiques commerciales telles que la publicité et la promotion.

Les réseaux sociaux sont des espaces originaux. Ils peuvent donner naissance à des compagnons de communication top-down conventionnels (un à plusieurs), mais aussi changer cette communication verticale et unilatérale vers un dialogue entre l'utilisateur et la marque (un à un) voire plus entre les internautes eux-mêmes (beaucoup à plusieurs).

Cependant, les consommateurs sont de moins en moins intéressés par la publicité traditionnelle, ce qui exerce une pression énorme sur les spécialistes du marketing. Le défi consiste à diffuser des informations complexes par le biais de messages sans submerger les consommateurs. L'attitude des consommateurs d'aujourd'hui a profondément changé, le temps d'appréciation et de lecture du message a beaucoup diminué. La durée d'attention moyenne des humains est passée de 12 secondes en 2000 à 8 secondes en 2013. Plus encore, cette attention est destinée à diminuer à l'avenir, en raison du volume massif et écrasant de messages bombardant constamment les appareils mobiles connectés qui nécessitent une attention immédiate des consommateurs (Kotler et al., 2017).

Les réseaux sociaux ont joué un rôle très important dans ces changements. Dans le passé, les consommateurs étaient limités aux publicités générées sur les médias traditionnels parce qu'ils n'avaient pas beaucoup de choix. Aujourd'hui, avec l'avènement des réseaux sociaux, les consommateurs disposent d'une richesse de contenus générés par des entreprises ou même par d'autres consommateurs qu'ils trouvent plus crédibles et intéressants. L'idée est que le contenu partagé sur les réseaux sociaux est volontaire et accessible à la demande, contrairement au contenu qui a été partagé sur les médias traditionnels dont le caractère est imposé.

$\mathrm{Au}$ niveau des médias sociaux, les consommateurs peuvent désormais choisir le contenu qu'ils découvrent et apprécient. Les publicités ne peuvent plus arrêter les téléspectateurs, comme c'est l'exemple des publicités YouTube (True View), qui précèdent les vidéos, les consommateurs peuvent désormais ignorer ces annonces après cinq secondes. Si les marques ou les annonceurs ne parviennent pas à attirer l'attention dans les cinq premières secondes, ils ne peuvent pas se plaindre que les consommateurs choisissent d'ignorer le reste de leur contenu (Kotler et al., 2017).

L'utilisation des réseaux sociaux, notamment via des stratégies de content marketing, est devenue très utile dans l'approche marketing des marques, d'abord en approchant favorablement les clients Internet de leurs marques grâce à un contact direct et immédiat. Ensuite, ils donnent aux marques le sens de l'innovation et le goût de la publicité, sans dépenser trop d'argent. Enfin, les réseaux sociaux permettent aux marques de bénéficier du bouche à oreille grâce aux interconnexions entre leurs membres. Les marques doivent être très à l'écoute des conseils, commentaires et publications laissés par les utilisateurs sur la page de la marque.

Malheureusement, les entreprises transfèrent souvent leur contenu sur les médias sociaux sans le modifier, étant donné que le contenu n'est qu'une version plus longue d'une publicité. Cependant, il existe une nette différence entre ces deux modes de communication, la publicité représente le message que la marque veut transmettre aux consommateurs. Alors que le marketing de contenu diffuse l'information que les consommateurs utiliseront pour atteindre leurs objectifs personnels et professionnels (Kotler et al., 2017).

Afin d'assurer une implication optimale des consommateurs, les entreprises doivent tirer parti des médias sociaux en créant des contenus qui, même s'ils ne contribuent pas à la vente et à la promotion de la marque, deviendront essentiels pour les consommateurs.

\section{Optimisation Pour les Moteurs de Recherche}

Une logique de marketing SEO (Search Engine Optimization) repose sur l'hypothèse que le trafic organique (trafic libre, sans publicité sur les médias) d'un site dépend de la qualité de son référencement 
naturel, de sorte que les moteurs de recherche (y compris, avant tout, le Google inégalé) peuvent le localiser et surtout le répertorier correctement (Sauvage, 2016).

Pour ce faire, de puissants algorithmes localisent le nom de domaine (un autre nom pour le site Web) et analysent le contenu. Ils identifient les liens tels que les liens internes et externes et les sources d'URL, et estiment la qualité de chaque page sur le sujet choisi. Cela suppose que le site Web est correctement structuré à la base afin que les robots puissent facilement passer d'une page à l'autre (Sauvage, 2016).

C'est pourquoi avoir une stratégie de contenu est pertinent d'un point de vue SEO : cela donne simplement plus de chances de dire à Google « voici de quoi je parle, et voici mon site avec les pages les plus pertinentes pour chaque sujet ».

La recette précise de chacun de ces algorithmes qui sont plus de 200, selon la firme de Mountain View reste un secret jalousement gardé. Cependant, au fil du temps, le référencement a été mieux compris par les spécialistes du marketing, et il a donc été partiellement démystifié.

\section{INBOUND MARKETING VS. MARKETING SORTANT}

Le marketing sortant est un marketing basé sur l'interruption, perçu comme le modèle traditionnel de promotion de produits dans lequel les gens doivent arrêter ce qu'ils font pour prêter attention au message marketing ou le traiter d'une autre manière (Rancati et al., 2015).

La différence entre l'inbound marketing et l'outbound marketing est constamment remise en question. L'inbound marketing a maintenant connu une croissance par rapport à l'outbound marketing qui est en déclin. En outre, les investissements dans les canaux de l'inbound marketing ont augmenté de manière significative, par rapport à d'autres canaux d'outbound marketing qui stagnent maintenant ou connaissent une croissance lente (Volpe, 2015).

Volpe (2015) suggère que la différence entre l'entrant et le sortant est artificielle et dénuée de sens. De plus, il propose un croisement entre les stratégies du marketing entrant et sortant, définies comme une zone grise (Volpe, 2015).

« L'Inbound Marketing crée top of mind preference, tandis que l'Outbound Marketing est principalement axé sur la Top of Mind Awareness. Avec les programmes de marketing de contenu entrant, vous pouvez aller au-delà de la simple sensibilisation pour générer des préférences et implicitement une dépendance à la marque, cet objectif peut être atteint grâce au positionnement d'une marque comme choix préféré » (Martin, 2013; Rancati et coll., 2015).

Le grand défi de l'Inbound Marketing est d'amener le consommateur à trouver l'entreprise en ligne, en raison de l'espace de mots clés très concurrentiel. Le délai dans lequel une entreprise attend que les consommateurs le trouvent en ligne peut entraîner une perte de vente (Rancati et al., 2015).

\section{CONCLUSION ET RECHERCHES FUTURES}

L'un des grands avantages d'Internet est qu'il atteint les consommateurs du monde entier, la mondialisation des affaires promet beaucoup grâce à l'avènement des nouvelles technologies.

L'inbound marketing est une stratégie qui vise à attirer des prospects sur le site Web d'une entreprise, à les convertir en prospects, puis en clients à vie et en promoteurs de marque.

Dans cet article, nous avons proposé une comparaison entre les stratégies Inbound et Outbound Marketing. Cela dit, sur la base des propositions de cet article, nous concluons que les stratégies Inbound et Outbound sont complémentaires pour le succès de toute campagne de marketing viable. Les deux stratégies ont leurs avantages et elles peuvent toutes deux être utilisées ensemble, souvent avec un effet plus important, créant une synergie.

En effet, le mélange de stratégies de marketing sortant et entrant peut fonctionner le plus efficacement et atteindre le plus grand nombre de personnes lorsqu'il est fait main dans la main.

Néanmoins, l'Inbound Marketing est avant tout du marketing de contenu et son efficacité est supérieure à l'efficacité de l'Outbound Marketing car l'inbound vise à identifier les clients cibles et à adapter la 
communication, cependant il permet un meilleur ciblage du public et une meilleure adaptation des mécanismes de communication de l'entreprise.

\section{Recherches Futures}

Cet article théorique peut être étendu pour englober une étude empirique qui calcule à travers différentes mesures l'impact des pratiques d'inbound marketing sur les consommateurs, via des données recueillies à partir de sites Web tels que www.Alexa.com.

\section{TRANSLATED VERSION: GERMAN}

Below is a rough translation of the insights presented above. This was done to give a general understanding of the ideas presented in the paper. Please excuse any grammatical mistakes and do not hold the original authors responsible for these mistakes.

\section{ÜBERSETZTE VERSION: DEUTSCH}

Hier ist eine ungefähre Übersetzung der oben vorgestellten Ideen. Dies wurde getan, um ein allgemeines Verständnis der in dem Dokument vorgestellten Ideen zu vermitteln. Bitte entschuldigen Sie alle grammatikalischen Fehler und machen Sie die ursprünglichen Autoren nicht für diese Fehler verantwortlich.

\section{EINLEITUNG}

Die Entwicklung der Marketingstrategie hat eine wertvolle Entwicklung durchlaufen, mit der Existenz der digitalen Kommunikation und der sozialen Netzwerke wurde die Erstellung von Inhalten geändert. Vor einigen Jahren nutzten die Unternehmen die traditionelle Strategie, um eine Botschaft an ein Publikum zu senden, um sie zu ihrem Unternehmen oder Produkt zu drängen, im Gegensatz zur Entwicklung der neuen Marketingstrategien, nämlich Inbound Marketing, eine neue Möglichkeit, das Publikum zum Unternehmen zu ziehen, indem innovativere Inhalte erstellt werden.

Der State of Inbound Marketing (Hubspot, 2013) beschreibt Outbound Marketing oder traditionelles Marketing als eine Technik, die von Unternehmen verwendet wird, um ein großes Publikum anzuziehen, in der Hoffnung, ein Feedback zu erhalten. Verwendung der Outbound-Strategie, die hauptsächlich auf der Verbreitung der Botschaft und der Werbung basiert, ohne das Ziel zu studieren, um eine effektive Antwort zu erhalten und mehr Umsatz und Einkommen zu generieren.

Outbound-Marketing ist unterbrechungsbasiertes Marketing, das als traditionelles Modell der Produktwerbung wahrgenommen wird, bei dem die Menschen aufhören müssen, was sie tun, um auf die Marketingbotschaft zu achten oder auf andere Weise damit umzugehen (Rancati et al., 2015). OutboundMarketing ist auch eine Strategie, bei der ein Unternehmen seine Produkte und Dienstleistungen bewirbt, indem es den Verbrauchern Informationen präsentiert, auch wenn sie nicht nach diesen Produkten oder Dienstleistungen suchen (Goodwin, 2013). Diese Strategie ist jedoch nicht mehr effektiv, um die Botschaft zu vermitteln und Traffic in der Webwelt zu generieren, weshalb Unternehmen heutzutage dazu neigen, neue Strategien wie den Inbound-Marketing-Ansatz zu erkunden, um mehr Ergebnisse aus dem Internet zu erzielen.

Im ersten zweiten Abschnitt dieses Papiers werden wir Inbound-Marketing definieren und einen Überblick über die Entstehung dieses Konzepts erhalten. Im dritten Abschnitt erläutern wir die Komponenten des Inbound-Marketings. Im vierten Abschnitt vergleichen wir Outbound- und InboundMarketing, um zu wissen, ob sie unabhängige oder komplementäre Strategien darstellen. 


\section{DEFINITIONEN VON INBOUND-MARKETING}

Inbound-Marketing ist die Strategie, sich mit potenziellen Kunden durch Materialien und Erfahrungen zu verbinden, die sie nützlich finden. Mit Medien wie Blogs und sozialen Netzwerken hoffen Vermarkter, die Zuschauer mit Inhalten zu unterhalten und zu informieren, die sie selbst suchen (Marketing-Schools.org, 2016).

Auf der anderen Seite kann Inbound-Marketing als eine Form des Marketings erklärt werden, die eine sehr sorgfältige Ausrichtung des Publikums und eine individuelle Kommunikation über qualitativ hochwertige Inhalte erfordert (Patrutiu-Baltes, 2016).

Darüber hinaus definierte Venisse (2016) Inbound-Marketing als eine Möglichkeit, zu einem eigenen Medium zu werden, damit das Publikum leichter in Kunden umgewandelt werden kann. Die Umsetzung der globalen Inbound-Marketing-Strategie erfordert die Einhaltung bestimmter wesentlicher Schritte (Venisse, 2016). Der erste Schritt besteht darin, eigene Medien zu entwickeln und ein qualifiziertes Publikum aufzubauen. Für ein Unternehmen, das diese Strategie aufstellt, ist es wichtig, interessante Inhalte auf seiner Website, seinem Blog oder seiner Social-Network-Seite anzubieten, um ein Publikum anzuziehen und zu erhalten.

Schwebel (2017) schlägt auch vor, dass Inbound-Marketing das Potenzial hat, eine Marke zu einem eigenen Medium zu entwickeln, das heißt, Inhalte anzubieten, die intelligent und relevant genug sind, um ein Publikum aufzubauen, anstatt aufdringliche Anzeigen (Dabi-Schwebel, 2017).

Cantin (2014) definiert Inbound-Marketing als eine digitale Marketingstrategie, die darauf abzielt, den Kunden in das Unternehmen zu bringen, anstatt ihn abzuholen. Der Interessent wird von relevanten Inhalten angezogen, die nach Reifephasen der Einkäufe und Engagementgraden positioniert sind. Daher könnten wir in dieser Gedankenlinie auch über das Akquisitionsmarketing sprechen (Cantin, 2014).

Wir definieren Inbound-Marketing auch als digitale Marketingstrategie, deren allgemeine Idee es ist, potenzielle Interessenten kontinuierlich mit qualitativ hochwertigen Inhalten anzuziehen. Dazu verwendet Inbound Marketing eine vierstufige Methodik: Attract, Convert, Transform und Retain (InboundValue.com, 2018).

Darüber hinaus kann Inbound-Marketing, wie Rouse (2014) vorschlug, "als eine Technik definiert werden, die sich darauf konzentriert, Verbraucher oder Leads über relevante Inhalte zu bringen, so dass Verbraucher nach dem Unternehmen suchen und nicht nach dem Gegenteil. Das Konzept des InboundMarketings erleichtert den Verbrauchern, die relevante Waren, Produkte oder Dienstleistungen wünschen, Aufgaben" (Rouse, 2014).

Hubspot (2018) definiert Inbound Marketing als eine Strategie, die Kunden mit relevanten und nützlichen Inhalten anzieht und bei jedem Schritt der Customer-Shopping-Journey einen Mehrwert schafft. Beim Inbound Marketing finden potenzielle Kunden ihren Weg über Kanäle wie Blogs, Suchmaschinen und Social Media (Hubspot, 2016). Im Gegensatz zu Outbound Marketing muss Inbound Marketing nicht um die Aufmerksamkeit potenzieller Kunden kämpfen. Durch die Erstellung von Inhalten, die auf die Probleme und Bedürfnisse idealer Kunden eingehen, zieht die Marke qualifizierte Interessenten an und baut Vertrauen und Glaubwürdigkeit auf. Daher ist Inbound-Marketing ein systemischer Ansatz, der es jeder Marke ermöglicht, ihre eigenen Medien zu werden, ein Publikum zu schaffen und es in Interessenten und dann in Kunden umzuwandeln. Ein ganzer Prozess funktioniert gut an den Details und eliminiert gleichzeitig jeden Fehler (Dabi-Schwebel, 2017).

\section{ENTSTEHUNG DES INBOUND MARKETINGS}

Der Begriff « Inbound » wurde von Brian Halligan verwendet (Halligan et al., 2009). Der Begriff Inbound bezieht sich auf die Gewinnung und Umwandlung von Einzelpersonen in dauerhafte Kunden.

In Anhnung an die Schöpfer des Inbound-Marketings Brian Halligan und Dhamesh Shah wurden die Bedürfnisse für dieses innovative Konzept aus der Tatsache geschaffen, dass die Menschen es satt haben, mit traditionellen Outbound-Marketing-Botschaften unterbrochen zu werden. Darüber hinaus führten die 
in der Vergangenheit erfolgreich eingesetzten Marketingtaktiken nicht mehr $\mathrm{zu}$ den gewünschten Ergebnissen (Halligan et al., 2009).

Alles begann, als Shah einen Blog über Startups und Unternehmertum startete, dann bemerkte er, dass sein Blog einen massiven Traffic mehr gewann als Websites von Unternehmen mit professionellen Marketing-Crews und großen Budgets. Nach vielen Analysen und Diskussionen kamen sie mit einer einfachen Beobachtung heraus, dass "die Menschen nicht von Vermarktern unterbrochen oder von Verkäufern belästigt werden wollten, ihnen will geholfen warden" (Halligan und Shah, 2014).

Die Hauptidee des Inbound-Marketings ist die Verwendung von Marketingtechniken, die versuchen, das Interesse der Kunden zu wecken, indem kreative und nützliche Inhalte geteilt werden, die über mehrere Online-Kanäle, einschließlich Suchmaschinen und soziale Medien, geteilt werden.

Auch wenn Inbound Marketing erstmals 2005 von Halligan und Shah eingeführt wurde, ist es eigentlich kein neues Konzept, es ähnelt der Marketingmethode namens "Content Marketing" und kann auch mit "Storytelling Marketing" in Verbindung gebracht werden, nämlich einer Technik, die lange vor der Entstehung des Internets verwendet wurde (Pulizzi, 2012).

Darüber hinaus wird Content-Marketing als Marketingprozess definiert, der darauf abzielt, relevante Inhalte zu entwickeln, um eine Zielgruppe anzuziehen und $\mathrm{zu}$ binden und so eine profitable Verbraucheraktion zu erhalten (Pulizzi, 2012).

Außerdem muss Content-Marketing strategische Planung, Content-Erstellung und Verteilung für mehrere Phasen des Kaufzyklus an mehrere Kunden umfassen, was bedeutet, dass eine vollständige Content-Marketing-Strategie das Inbound-Marketing-Prinzip beinhalten sollte (Pulizzi, 2011).

Eine der Techniken des Inbound-Marketings, die als Suchmaschinenoptimierung (SEO) bekannt ist, wurde lange Vor dem Konzept des Content-Marketings praktiziert, und SEO wird hauptsächlich verwendet, um die Anzahl der Besucher zu erhöhen, die von Suchmaschinen kommen.

Die Keywords galten als das wichtigste Element, um ein hohes Ranking zu erreichen, dann nahm mit der Zeit und nach der Einführung vieler Updates, die enthalten waren, wie Panda im Jahr 2011, Penguin im Jahr 2012 und das Hummingbird-Update im Jahr 2013 (Wainwright, 2014), diese Bedeutung von Keywords ab. Aufgrund dieser Updates wird SEO auch zu einer sehr schwierigen Aufgabe. Von hier aus kommt Inbound-Marketing an Ort und Stelle, laut Rand Fishkin ist SEO eine Taktik und Inbound-Marketing ist eine Strategie.

E-Mail-Marketing bezieht sich auch auf eines der wichtigen Elemente des Inbound-Marketings, das auf dem Versand von Newslettern, Artikeln oder anderen über eine E-Mail basiert. Es ist eine Art von Marketing, die auf der Erlaubnis der Besucher basiert, ist es auch als Permission Marketing bekannt, nämlich ein Konzept, das von Seth Godin (Godin, 1999) entwickelt wurde.

\section{KOMPONENTEN DES INBOUND-MARKETINGS}

\section{Content Marketing}

Obwohl Content Marketing angesichts des damaligen Mangels an Technologie im Schatten des traditionellen Marketings geblieben ist, hat es sich heute geschafft, sich insbesondere mit dem Aufkommen neuer Technologien als effektive Strategie zu etablieren.

Chaffey und Al (2000) definieren Inhalt als ein Text- und Grafikinformationsdesign, das eine Webseite bildet. Guter Inhalt ist ein Grundpfstein, um Kunden auf eine Website zu locken und ihr Interesse zu behalten, während wiederholte Besuche provoziert werden. Content Marketing kann auch der IntranetInhalt des Unternehmens oder der Internet-Inhalt des Unternehmens sein, einschließlich Datenbanken und verschiedener Arten von Wissen (Chaffey und Al, 2000).

Pulizzi und Barret (2009) definieren Content Marketing auch als eine Marketingtechnik, die darin besteht, relevante, nützliche und wertvolle Inhalte zu erstellen, zu veröffentlichen und zu verteilen, um Gewinne von den Verbrauchern zu erzielen. Content-Marketing neigt dazu, Medien zu haben, anstatt sie zu mieten. Es ist definiert als ein Marketingprozess, der Kunden anzieht und hält, indem er ständig Inhalte erstellt, die darauf abzielten, das Verbraucherverhalten zu ändern oder weiterzuentwickeln (Pulizzi, J. und Barrett, 2009). 
Handley und Chapman (2010) argumentieren, dass Content Marketing alles ist, was eine Person oder Organisation erstellt oder teilt, um ihre Geschichten zu erzählen. Der Inhalt ist dialogisch, menschlich und versucht nicht ständig zu verkaufen. Es muss ein Geisteszustand sein, der sowohl schüchtern als auch ermutigt ist (Handley und Chapman, 2010).

Gunelius (2011) definiert Content Marketing als einen Prozess der direkten und indirekten Förderung eines Unternehmens oder einer Marke durch den Nutzen eines Text-, Video- oder Audioinhalts gleichzeitig in Online- und Offline-Umgebungen. Es kann in Langform (Blogs, Artikel, E-Books und Sound), in Kurzform (Twitter, Facebook, Bilder ...) oder in herkömmlicher Form (Teilen von Inhalten über Twitter oder Teilnahme an einer aktiven Diskussion über Kommentare zu Blogbeiträgen oder über Online-Foren) erscheinen (Gunelius, 2011).

Staler (2014) erklärt, dass Content-Marketing eine dynamische Technik ist, die darauf abzielt, Kunden anzuziehen, es ist ein Prozess des Austauschs von Fachwissen und Wissen, um potenzielle Kunden anzuziehen und eine Beziehung zu ihnen aufzubauen (Slater, 2014).

Ramos (2014) definiert Content Marketing als eine qualitativ hochwertige Marketingtechnik, relevante Inhalte, die darauf abzielen, lehrreich und mobilisierend zu sein, um die Zielgruppe zu erreichen (Ramos, 2014).

Kotler et al. (2017) definieren Content Marketing als einen Marketingansatz, der darin besteht, interessante, relevante und nützliche Inhalte für eine klar definierte Zielgruppengruppe zu erstellen, zu organisieren, zu verteilen und zu verstärken, um Content-Konversationen zu erstellen. Content Marketing ist auch eine weitere Form von Branding und Markenjournalismus, die engere Verbindungen zwischen Marken und Kunden schafft. Letztendlich geht es beim Content Marketing darum, die Leistung für eine Zielgruppe $\mathrm{zu}$ planen, zu erstellen, zu fördern und $\mathrm{zu}$ überwachen, immer mit dem Ziel der Kundenzufriedenheit und Umsatzgenerierung (Kotler et al., 2017).

\section{Social Media Marketing}

Die Eigenschaften von Social Media und ihre immense Popularität haben Geschäftspraktiken wie Werbung und Promotion revolutioniert.

Soziale Netzwerke sind originelle Räume. Sie können zu herkömmlichen Top-DownKommunikationsbegleitern (einer $\mathrm{zu}$ vielen) führen, aber auch diese vertikale und einseitige Kommunikation in Richtung eines Dialogs zwischen dem Nutzer und der Marke (eins zu eins) oder sogar mehr zwischen den Internetnutzern selbst (viele zu vielen) verändern.

Die Verbraucher interessieren sich jedoch immer weniger für traditionelle Werbung, was einen enormen Druck auf die Vermarkter ausübt. Die Herausforderung besteht darin, komplexe Informationen durch Nachrichten zu verbreiten, ohne die Verbraucher zu überfordern. Die Einstellung der heutigen Verbraucher hat sich tiefgreifend verändert, die Zeit der Wertschätzung und des Lesens von Botschaften hat stark abgenommen. Die durchschnittliche Aufmerksamkeitsspanne des Menschen ist von 12 Sekunden im Jahr 2000 auf 8 Sekunden im Jahr 2013 gesunken. Mehr noch, diese Aufmerksamkeit wird in Zukunft abnehmen, da die massive und überwältigende Menge an Nachrichten ständig vernetzte mobile Geräte bombardiert, die sofortige Aufmerksamkeit der Verbraucher erfordern (Kotler et al., 2017).

Soziale Netzwerke haben bei diesen Veränderungen eine sehr wichtige Rolle gespielt. In der Vergangenheit waren die Verbraucher auf Werbespots beschränkt, die auf traditionellen Medien generiert wurden, weil sie nicht viel Auswahl hatten. Heute, mit dem Aufkommen sozialer Netzwerke, haben die Verbraucher eine Fülle von Inhalten, die von Unternehmen oder sogar von anderen Verbrauchern generiert werden, die sie glaubwürdiger und interessanter finden. Die Idee ist, dass Inhalte, die in sozialen Netzwerken geteilt werden, freiwillig und auf Abruf zugänglich sind, im Gegensatz zu Inhalten, die in traditionellen Medien geteilt wurden, deren Charakter auferlegt wird.

Auf der Social-Media-Ebene können Verbraucher nun wählen, welche Inhalte sie entdecken und genießen. Anzeigen können Zuschauer nicht mehr stoppen, wie es das Beispiel von YouTube-Anzeigen (True View) ist, die Videos vorausgehen, Verbraucher können diese Anzeigen nun nach fünf Sekunden ignorieren. Wenn Marken oder Werbetreibende in den ersten fünf Sekunden nicht auffallen, können sie sich nicht darüber beschweren, dass Verbraucher den Rest ihrer Inhalte ignorieren (Kotler et al., 2017). 
Die Nutzung sozialer Netzwerke, insbesondere über Content-Marketing-Strategien, ist im Marketingansatz von Marken sehr nützlich geworden, indem sie zunächst Internetkunden ihrer Marken dank des direkten und sofortigen Kontakts günstig ansprechen. Dann geben sie Marken das Gefühl von Innovation und den Geschmack von Werbung, ohne zu viel Geld auszugeben. Schließlich ermöglichen soziale Netzwerke Marken, durch die Verbindungen zwischen ihren Mitgliedern von Mundpropaganda zu profitieren. Marken müssen sehr aufmerksam sein und auf Ratschläge, Kommentare und Veröffentlichungen hören, die von Benutzern auf der Seite der Marke hinterlassen werden.

Leider übertragen Unternehmen ihre Inhalte oft in soziale Medien, ohne sie zu ändern, wenn man bedenkt, dass Inhalte nur eine längere Version einer Werbung sind. Es gibt jedoch einen klaren Unterschied zwischen diesen beiden Kommunikationsmodi, Werbung repräsentiert die Botschaft, die die Marke den Verbrauchern vermitteln möchte. Während Content-Marketing die Informationen verbreitet, die Verbraucher verwenden werden, um ihre persönlichen und beruflichen Ziele zu erreichen (Kotler et al., 2017).

Um eine optimale Beteiligung der Verbraucher zu gewährleisten, müssen Unternehmen die sozialen Medien nutzen, indem sie Inhalte erstellen, die, auch wenn sie nicht zum Verkauf und zur Förderung der Marke beitragen, die Marke für die Verbraucher unerlässlich werden.

\section{Suchmaschinenoptimierung}

Eine SEO-Marketinglogik (Search Engine Optimization) basiert auf der Annahme, dass der organische Traffic (kostenloser Traffic, ohne Werbung auf Medien) einer Website von der Qualität ihrer natürlichen Referenzierung abhängt, so dass Suchmaschinen (einschließlich in erster Linie des unübertroffenen Google) sie finden und vor allem korrekt auflisten können (Sauvage, 2016).

Dazu lokalisieren leistungsfähige Algorithmen den Domainnamen (ein anderer Name für die Website) und analysieren den Inhalt. Sie identifizieren Links wie interne und externe Links und URL-Quellen und schätzen die Qualität jeder Seite zum gewählten Thema. Dies setzt voraus, dass die Website an der Basis richtig strukturiert ist, so dass die Bots leicht von einer Seite zur anderen wechseln können (Sauvage, 2016).

Aus diesem Grund ist eine Content-Strategie aus SEO-Sicht relevant: Sie gibt einfach mehr Chancen, Google zu sagen: "Hier ist, worüber ich spreche, und hier ist meine Website mit den relevantesten Seiten für jedes Thema".

Das genaue Rezept für jeden dieser Algorithmen, die laut der Firma Mountain View mehr als 200 sind, bleibt ein eifersüchtig gehütetes Geheimnis. Im Laufe der Zeit wurde SEO jedoch von Vermarktern besser verstanden und daher teilweise entlarvt.

\section{INBOUND MARKETING VS. OUTBOUND-MARKETING}

Outbound-Marketing ist unterbrechungsbasiertes Marketing, das als traditionelles Modell der Produktwerbung wahrgenommen wird, bei dem die Menschen aufhören müssen, was sie tun, um auf die Marketingbotschaft zu achten oder auf andere Weise damit umzugehen (Rancati et al., 2015).

Der Unterschied zwischen Inbound- und Outbound-Marketing wird ständig in Frage gestellt. InboundMarketing hat jetzt ein Wachstum im Vergleich zum Outbound-Marketing erlebt, das rückläufig ist. Darüber hinaus sind die Investitionen in die Kanäle des Inbound-Marketings im Vergleich zu anderen Kanälen des Outbound-Marketings, die jetzt entweder stagnieren oder ein langsames Wachstum verzeichnen, deutlich gestiegen (Volpe, 2015).

Volpe (2015) schlägt vor, dass der Unterschied zwischen dem Eingehenden und dem Ausgehenden künstlich und bedeutungslos ist. Darüber hinaus schlägt er eine Kreuzung zwischen den Strategien des Incoming- und Outgoing-Marketings vor, definiert als Grauzone (Volpe, 2015).

"Inbound-Marketing schafft Top-of-Mind-Präferenzen, während Outbound-Marketing sich hauptsächlich auf Top of Mind Awareness konzentriert. Mit Inbound-Content-Marketing-Programmen können Sie über einfache Bekanntheit hinausgehen, um Präferenzen und implizit Markensucht zu erzeugen, dieses Ziel kann durch die Positionierung einer Marke als bevorzugte Wahl erreicht warden" (Martin, 2013; Rancati et al., 2015). 
Die große Herausforderung für Inbound Marketing besteht darin, den Verbraucher dazu zu bringen, das Unternehmen online zu finden, da der Keyword-Raum hart umkämpft ist. Der Zeitrahmen, in dem ein Unternehmen darauf wartet, dass Verbraucher es online finden, kann zu einem Verkaufsverlust führen (Rancati et al., 2015).

\section{FAZIT UND ZUKÜNFTIGE FORSCHUNG}

Einer der großen Vorteile des Internets ist, dass es Verbraucher auf der ganzen Welt erreicht, die Globalisierung der Wirtschaft verspricht viel dank des Aufkommens neuer Technologien.

Das Inbound-Marketing ist eine Strategie, die darauf abzielt, Interessenten für die Website eines Unternehmens zu gewinnen, sie in Leads und dann weiter zu lebenslangen Kunden und Markenpromotern zu konvertieren.

In diesem Artikel haben wir einen Vergleich zwischen Inbound- und Outbound-Marketingstrategien vorgeschlagen. Basierend auf den Vorschlägen dieses Papiers kommen wir jedoch zu dem Schluss, dass sowohl Inbound- als auch Outbound-Strategien den Erfolg jeder tragfähigen Marketingkampagne ergänzen. Beide Strategien haben ihre Vorteile und sie können beide zusammen verwendet werden, oft mit einer größeren Wirkung, wodurch eine Synergie entsteht.

In der Tat kann die Mischung aus Outbound- und Inbound-Marketingstrategien am effektivsten funktionieren und die meisten Menschen erreichen, wenn sie Hand in Hand durchgeführt werden.

Dennoch ist Inbound Marketing in erster Linie Content Marketing und seine Effizienz ist der Outbound Marketing Effizienz überlegen, da Inbound darauf abzielt, die Zielkunden zu identifizieren und die Kommunikation anzupassen, aber es ermöglicht eine bessere Ausrichtung der Zielgruppe und eine bessere Anpassung der Kommunikationsmechanismen des Unternehmens.

\section{Zukünftige Forschung}

Dieser theoretische Artikel kann um eine empirische Studie erweitert werden, die anhand verschiedener Metriken die Auswirkungen von Inbound-Marketingpraktiken auf Verbraucher über Daten berechnet, die von Websites wie www.Alexa.com gesammelt werden.

\section{TRANSLATED VERSION: PORTUGUESE}

Below is a rough translation of the insights presented above. This was done to give a general understanding of the ideas presented in the paper. Please excuse any grammatical mistakes and do not hold the original authors responsible for these mistakes.

\section{VERSÃO TRADUZIDA: PORTUGUÊS}

Aqui está uma tradução aproximada das ideias acima apresentadas. Isto foi feito para dar uma compreensão geral das ideias apresentadas no documento. Por favor, desculpe todos os erros gramaticais e não responsacule os autores originais responsáveis por estes erros.

\section{INTRODUÇÃO}

O desenvolvimento da estratégia de marketing passou por uma evolução valiosa, com a existência da comunicação digital e das redes sociais, a criação de conteúdo foi alterada. Há alguns anos, as empresas utilizavam a estratégia tradicional, como enviar uma mensagem para o público, a fim de empurrá-las para sua empresa ou produto, em frente ao desenvolvimento das novas estratégias de marketing, ou seja, o Inbound Marketing, uma nova forma de atrair o público para a empresa, criando conteúdos mais inovadores.

O State of Inbound Marketing (Hubspot, 2013) descreve o Outbound Marketing ou o marketing tradicional como uma técnica usada pelas empresas para atrair um grande público, na esperança de obter 
um feedback. Utilizando a estratégia de saída, principalmente baseada na difusão da mensagem e publicidade, sem estudar a meta, a fim de receber uma resposta efetiva e gerar mais vendas e renda.

O outbound marketing é o marketing baseado em interrupção, percebido como o modelo tradicional de promoção de produtos em que as pessoas têm que parar o que estão fazendo para prestar atenção à mensagem de marketing ou lidar com ela de alguma outra forma (Rancati et al., 2015). O outbound marketing também é uma estratégia na qual uma empresa anuncia seus produtos e serviços, apresentando informações aos consumidores mesmo que não estejam procurando por esses produtos ou serviços (Goodwin, 2013). No entanto, essa estratégia não é mais eficaz em passar a mensagem e fazer o tráfego no mundo web, por isso as empresas, hoje em dia, tendem a explorar novas estratégias, como a abordagem inbound marketing, a fim de obter mais resultados da internet.

Na primeira segunda seção deste artigo, definiremos o inbound marketing e teremos uma visão geral do surgimento desse conceito. Na terceira seção, explicaremos os componentes do inbound marketing. Na quarta seção, vamos comparar Outbound e Inbound Marketing para saber se eles representam estratégias independentes ou complementares.

\section{DEFINIÇÕES DE INBOUND MARKETING}

O inbound marketing é a estratégia de se conectar com potenciais clientes através de materiais e experiências que eles acham úteis. Usando mídias como blogs e redes sociais, os profissionais de marketing esperam entreter e informar os espectadores com conteúdo que buscam por si mesmos (MarketingSchools.org, 2016).

Por outro lado, o inbound marketing pode ser explicado como uma forma de marketing que requer um direcionamento muito cuidadoso do público e uma comunicação personalizada via conteúdo de alta qualidade (Patrutiu-Baltes, 2016).

Além disso, a Venisse (2016) definiu o inbound marketing como uma forma de se tornar sua própria mídia para que o público se converta mais facilmente em clientes. A implementação da estratégia global de inbound marketing requer o respeito de certas etapas essenciais (Venisse, 2016). O primeiro passo é desenvolver sua própria mídia e construir um público qualificado. Para uma empresa que monta essa estratégia, é importante oferecer conteúdo interessante em seu site, seu blog ou sua página em rede social, para atrair e sustentar uma audiência.

Além disso, Schwebel (2017) sugere que o inbound marketing tem o potencial de desenvolver uma marca para se tornar seu próprio meio, ou seja, oferecer conteúdo inteligente e relevante o suficiente para construir uma audiência, em vez de anúncios intrusivos (Dabi-Schwebel, 2017).

Cantin (2014) define que o inbound marketing é uma estratégia de marketing digital que visa trazer o cliente para a empresa em vez de pegá-lo. A perspectiva é atraída por conteúdo relevante posicionado de acordo com fases de vencimento de compras e níveis de comprometimento. Portanto, nessa linha de pensamentos, poderíamos também falar sobre o marketing de aquisição (Cantin, 2014).

Definimos o inbound marketing também como uma estratégia de marketing digital cuja ideia geral é atrair continuamente potenciais perspectivas com conteúdo de qualidade. Para isso, a Inbound Marketing utiliza uma metodologia de quatro etapas: Atrair, Converter, Transformar e Reter (InboundValue.com, 2018).

Além disso, como propôs Rouse (2014), o inbound marketing pode ser definido "como uma técnica que se concentra em trazer consumidores ou leads via conteúdo relevante, para que os consumidores busquem a empresa em vez do contrário. O conceito de inbound marketing facilita tarefas para os consumidores que querem bens, produtos ou serviços relevantes" (Rouse, 2014).

A Hubspot (2018) define o Inbound Marketing como uma estratégia que atrai clientes com conteúdo relevante e útil e agrega valor a cada etapa da jornada de compra do cliente. Com o inbound marketing, os potenciais clientes encontram seu caminho através de canais como blogs, mecanismos de busca e mídias sociais (Hubspot, 2016). Ao contrário do Outbound Marketing, o Inbound Marketing não precisa lutar pela atenção de potenciais clientes. Ao criar conteúdo que atenda aos problemas e necessidades dos clientes ideais, a marca atrai perspectivas qualificadas e constrói confiança e credibilidade. Assim, o inbound 
marketing é uma abordagem sistêmica que permite que cada marca se torne sua própria mídia, crie uma audiência e converta-a em prospects e, em seguida, clientes. Todo um processo funciona bem nos detalhes enquanto elimina qualquer falha (Dabi-Schwebel, 2017).

\section{SURGIMENTO DO MARKETING DE ENTRADA}

O termo «Entrada» foi usado por Brian Halligan (Halligan et al., 2009). O termo entrada refere-se à atração e conversão de indivíduos para clientes duradouros.

Correspondente aos criadores do inbound marketing Brian Halligan e Dhamesh Shah, as necessidades para este conceito inovador foram criadas a partir do fato de que as pessoas estão doentes e cansadas de serem interrompidas com mensagens tradicionais de marketing de saída. Além disso, as táticas de marketing usadas com sucesso no passado não produziram mais os resultados do desejo (Halligan et al., 2009).

Tudo começou quando Shah começou um blog sobre startups e empreendedorismo, então, ele notou que seu blog ganhou um tráfego enorme mais do que sites de propriedade de empresas com equipes de marketing profissionais e grandes orçamentos. Depois de muitas análises e discussões, eles saíram com uma simples observação de que "As pessoas não queriam ser interrompidas por profissionais de marketing ou assediadas por vendedores, elas querem ser ajudadas" (Halligan e Shah, 2014).

A ideia principal do inbound marketing é usar técnicas de marketing que tentarão captar o interesse dos clientes, compartilhando conteúdo criativo e útil que é compartilhado através de vários canais online, incluindo mecanismos de busca e mídias sociais.

Mesmo que o inbound marketing tenha sido introduzido pela primeira vez em 2005 por Halligan e Shah, na verdade não é um conceito novo, é semelhante ao método de marketing chamado "Marketing de Conteúdo" e também pode ser associado ao "Marketing de Storytelling", ou seja, uma técnica usada antes do surgimento da internet (Pulizzi, 2012).

Além disso, o marketing de conteúdo é definido como um processo de marketing que visa desenvolver conteúdo relevante a fim de atrair e engajar um público-alvo, obtendo assim uma ação lucrativa do consumidor (Pulizzi, 2012).

Além disso, o marketing de conteúdo deve incluir planejamento estratégico, criação de conteúdo e distribuição para múltiplas etapas do ciclo de compra para vários clientes, o que significa que uma estratégia completa de marketing de conteúdo deve incorporar o princípio de inbound marketing (Pulizzi, 2011).

Uma das técnicas de inbound marketing conhecida como otimização do mecanismo de busca (SEO) foi praticada por muito tempo antes do conceito de marketing de conteúdo, e o SEO é usado principalmente para aumentar o número de visitantes que vêm de mecanismos de busca.

As palavras-chave foram consideradas o elemento-chave para alcançar o alto escalão, então, com o tempo e após a introdução de muitas atualizações que foram incluídas, como Panda em 2011, Penguin em 2012 e a atualização do Beija-Flor em 2013 (Wainwright, 2014), essa importância das palavras-chave diminuiu. Além disso, por causa dessas atualizações, o SEO se torna uma tarefa muito difícil. A partir daqui, o inbound marketing vem no lugar, de acordo com Rand Fishkin, o SEO é uma tática, e o inbound marketing é uma estratégia.

O e-mail marketing também se refere a um dos elementos importantes do inbound marketing com base no envio de boletins informativos, artigos ou outros, por meio de um e-mail. É um tipo de marketing baseado na permissão dada pelos visitantes, é também conhecido como marketing de permissão, ou seja, um conceito que foi desenvolvido por Seth Godin (Godin, 1999).

\section{COMPONENTES DO MARKETING DE ENTRADA}

\section{Marketing de Conteúdo}

Embora o marketing de conteúdo tenha permanecido à sombra do marketing tradicional dada a falta de tecnologia na época, ele tem conseguido hoje estabelecer-se como uma estratégia eficaz, especialmente com o surgimento de novas tecnologias. 
Chaffey e $\mathrm{Al}$ (2000) definem o conteúdo como um design de informações gráficas e texto que forma uma página da Web. Um bom conteúdo é uma pedra fundamental para atrair clientes para um site e manter seu interesse enquanto provoca visitas repetidas. O marketing de conteúdo também pode ser o conteúdo intranet da empresa ou o conteúdo da empresa na internet, incluindo bancos de dados e vários tipos de conhecimento (Chaffey e Al, 2000).

Por sua vez, Pulizzi e Barret (2009) também definem o marketing de conteúdo como uma técnica de marketing que consiste em criar, publicar e distribuir conteúdo relevante, útil e valioso para obter lucros dos consumidores. O marketing de conteúdo tende a ter mídia em vez de alugá-la. É definido como um processo de marketing que atrai e retém clientes, criando constantemente conteúdo que visa mudar ou evoluir o comportamento do consumidor (Pulizzi, J. e Barrett, 2009).

Handley e Chapman (2010) argumentam que o marketing de conteúdo é tudo o que um indivíduo ou organização cria ou compartilha para contar suas histórias. O conteúdo é conversador, humano e não busca constantemente vender. Deve ser um estado de espírito tímido e encorajado (Handley e Chapman, 2010).

Gunelius (2011) define o marketing de conteúdo como um processo de promoção direta e indireta de uma empresa ou marca através do benefício de um conteúdo de texto, vídeo ou áudio por vez, em ambientes online e offline. Ele pode aparecer de forma longa (blogs, artigos, e-books e som), em forma curta (Twitter, Facebook, imagens...), ou de forma convencional, (compartilhamento de conteúdo via Twitter ou participar de uma discussão ativa através de comentários em posts de blog ou através de fóruns online) (Gunelius, 2011).

Staler (2014) explica que o marketing de conteúdo é uma técnica dinâmica voltada para atrair clientes, é um processo de compartilhamento de expertise e conhecimento a fim de atrair potenciais clientes e estabelecer um relacionamento com eles (Slater, 2014).

Ramos (2014) define o marketing de conteúdo como uma técnica de marketing de alta qualidade, conteúdo relevante que visa ser educativo e mobilizador para adquirir o público-alvo (Ramos, 2014).

Kotler et al. (2017) definem o marketing de conteúdo como uma abordagem de marketing que consiste em criar, organizar, distribuir e amplificar conteúdo interessante, relevante e útil para um grupo de público claramente definido para criar conversas de conteúdo. O marketing de conteúdo também é outra forma de branding e jornalismo de marca que cria laços mais próximos entre marcas e clientes. Em última análise, o marketing de conteúdo é sobre planejamento, criação, promoção e monitoramento do desempenho para um público-alvo, sempre com o objetivo de satisfação do cliente e geração de receita em mente (Kotler et al., 2017).

\section{Marketing de Mídia Social}

As características das mídias sociais e sua imensa popularidade revolucionaram práticas comerciais como publicidade e promoção.

Redes sociais são espaços originais. Eles podem dar origem aos companheiros convencionais de comunicação de cima para baixo (um para muitos), mas também mudar essa comunicação vertical e unilateral para um diálogo entre o usuário e a marca (um a um) ou até mais entre os próprios usuários da Internet (muitos para muitos).

No entanto, os consumidores estão cada vez menos interessados na publicidade tradicional, o que coloca enorme pressão sobre os profissionais de marketing. O desafio está em espalhar informações complexas através de mensagens sem sobrecarregar os consumidores. A atitude dos consumidores de hoje mudou profundamente, o tempo de apreciação e leitura de mensagens diminuiu muito. O tempo médio de atenção dos humanos diminuiu de 12 segundos em 2000 para 8 segundos em 2013. Ainda mais, essa atenção está destinada a diminuir no futuro, devido ao enorme e avassalador volume de mensagens constantemente bombardeando dispositivos móveis conectados que requerem atenção imediata do consumidor (Kotler et al., 2017).

As redes sociais têm desempenhado um papel muito importante nessas mudanças. No passado, os consumidores se limitavam aos comerciais gerados na mídia tradicional porque não tinham muita escolha. Hoje, com o advento das redes sociais, os consumidores têm uma riqueza de conteúdo gerado por empresas ou mesmo por outros consumidores que acham mais críveis e interessantes. A ideia é que o conteúdo que 
é compartilhado nas redes sociais seja voluntário e acessível sob demanda, ao contrário do conteúdo que foi compartilhado na mídia tradicional cujo caráter é imposto.

No nível das mídias sociais, os consumidores agora podem escolher qual conteúdo descobrem e desfrutam. Os anúncios não podem mais parar os espectadores, pois é o exemplo de anúncios do YouTube (True View), que precedem vídeos, os consumidores agora podem ignorar esses anúncios após cinco segundos. Se marcas ou anunciantes não conseguirem chamar a atenção nos primeiros cinco segundos, eles não podem reclamar que os consumidores optam por ignorar o resto de seu conteúdo (Kotler et al., 2017).

O uso de redes sociais, especialmente por meio de estratégias de marketing de conteúdo, tornou-se muito útil na abordagem de marketing das marcas, primeiro por aproximar-se de clientes da Internet de suas marcas favoravelmente graças ao contato direto e imediato. Então, dão às marcas a sensação de inovação e gosto da publicidade, sem gastar muito dinheiro. Por fim, as redes sociais permitem que as marcas se beneficiem do boca a boca através das interconexões entre seus membros. As marcas devem estar muito atentas e ouvir conselhos, comentários e publicações deixados pelos usuários na página da marca.

Infelizmente, muitas vezes as empresas transferem seu conteúdo para mídias sociais sem modificá-lo, considerando que o conteúdo é apenas uma versão mais longa de um anúncio. No entanto, há uma clara diferença entre esses dois modos de comunicação, a publicidade representa a mensagem que a marca quer transmitir aos consumidores. Enquanto o marketing de conteúdo divulga as informações que os consumidores usarão para alcançar seus objetivos pessoais e profissionais (Kotler et al., 2017).

Para garantir o envolvimento ideal do consumidor, as empresas devem aproveitar as mídias sociais criando conteúdos que, mesmo que não contribuam para a venda e promoção da marca, a marca se tornará essencial para os consumidores.

\section{Otimização do Mecanismo de Busca}

Uma lógica de marketing de SEO (Search Engine Optimization) baseia-se no pressuposto de que o tráfego orgânico (tráfego livre, sem publicidade na mídia) de um site depende da qualidade de seu referenciamento natural, para que os mecanismos de busca (incluindo, em primeiro lugar, o Inigualável Google) possam localizá-lo e listá-lo corretamente (Sauvage, 2016).

Para isso, algoritmos poderosos localizam o nome de domínio (outro nome para o site) e analisam o conteúdo. Eles identificam a vinculação, como links internos e externos e fontes de URL, e estimam a qualidade de cada página sobre o tópico escolhido. Isso pressupõe que o site esteja devidamente estruturado na base para que os bots possam facilmente ir de uma página para outra (Sauvage, 2016).

É por isso que ter uma estratégia de conteúdo é relevante do ponto de vista do SEO: ele simplesmente dá mais chances de dizer ao Google "aqui está o que estou falando, e aqui está meu site com as páginas mais relevantes para cada assunto".

A receita precisa para cada um desses algoritmos que são mais de 200, de acordo com a empresa de Mountain View continua sendo um segredo zeloso guardado. No entanto, ao longo do tempo, o SEO tem sido melhor compreendido pelos profissionais de marketing, e por isso foi parcialmente desmascarado.

\section{INBOUND MARKETING VS. MARKETING DE SAÍDA}

O outbound marketing é o marketing baseado em interrupção, percebido como o modelo tradicional de promoção de produtos em que as pessoas têm que parar o que estão fazendo para prestar atenção à mensagem de marketing ou lidar com ela de alguma outra forma (Rancati et al., 2015).

A diferença entre o marketing de entrada e saída é constantemente desafiada. O inbound marketing já experimentou crescimento em comparação com o outbound marketing que está em declínio. Além disso, os investimentos nos canais de inbound marketing passaram a aumentar significativamente, em comparação

com outros canais de outbound marketing que agora estão estagnados ou estão experimentando crescimento lento (Volpe, 2015).

Volpe (2015) sugere que a diferença entre a entrada e a saída é artificial e sem sentido. Além disso, propõe um cruzamento entre as estratégias do marketing de entrada e saída, definido como zona cinzenta (Volpe, 2015). 
"O Inbound Marketing cria a Preferência do Topo da Mente, enquanto o Outbound Marketing é focado principalmente no Top of Mind Awareness. Com programas de marketing de conteúdo de entrada, você pode ir além da simples consciência para gerar preferência e vício implicitamente da marca, esse objetivo pode ser alcançado através do posicionamento de uma marca como a escolha preferida" (Martin, 2013; Rancati et al., 2015).

O grande desafio da Inbound Marketing é fazer com que o consumidor encontre a empresa online, por causa do espaço de palavras-chave altamente competitivo. O prazo em que uma empresa está esperando que os consumidores a encontrem online pode resultar em uma perda de venda (Rancati et al., 2015).

\section{CONCLUSÃO E FUTURA PESQUISA}

Uma das grandes vantagens da internet é que ela atinge consumidores em todo o mundo, a globalização dos negócios promete muito graças ao advento de novas tecnologias.

O inbound marketing é uma estratégia que visa atrair potenciais para o site de uma empresa, convertêlos em leads e, em seguida, mais longe, para clientes ao longo da vida e promotores de marcas.

Neste artigo, propusemos uma comparação entre as estratégias de Inbound e Outbound Marketing. Dito isto, com base nas propostas deste artigo, concluímos que tanto as estratégias de Entrada quanto de Saída são complementares para o sucesso de qualquer campanha de marketing viável. Ambas as estratégias têm seus profissionais e ambas podem ser usadas juntas, muitas vezes para um efeito maior, criando uma sinergia.

De fato, a mistura de estratégias de outbound e inbound marketing pode funcionar de forma mais eficaz e atingir a maior quantidade de pessoas quando feito de mãos dadas

No entanto, o Inbound Marketing é principalmente marketing de conteúdo e sua eficiência é superior à eficiência do Outbound Marketing, pois a entrada visa identificar os clientes-alvo e adaptar a comunicação, porém permite um melhor direcionamento do público e uma melhor adaptação dos mecanismos de comunicação da empresa.

\section{Pesquisa Futura}

Este artigo teórico pode ser estendido para englobar um estudo empírico que calcula por meio de diferentes métricas o impacto das práticas de inbound marketing nos consumidores, através de dados coletados de sites como www.Alexa.com. 